TRANSACTIONS OF THE

AMERICAN MATHEMATICAL SOCIETY

Volume 359, Number 10, October 2007, Pages 4669-4694

S 0002-9947(07)04008-1

Article electronically published on May 1, 2007

\title{
POISSON PI ALGEBRAS
}

\author{
S. P. MISHCHENKO, V. M. PETROGRADSKY, AND A. REGEV
}

\begin{abstract}
We study Poisson algebras satisfying polynomial identities. In particular, such algebras satisfy "customary" identities (Farkas, 1998, 1999) Our main result is that the growth of the corresponding codimensions of a Poisson algebra with a nontrivial identity is exponential, with an integer exponent. We apply this result to prove that the tensor product of Poisson PI algebras is a PI-algebra. We also determine the growth of the Poisson-Grassmann algebra and of the Hamiltonian algebras $\mathbf{H}_{2 k}$.
\end{abstract}

\section{Introduction: Poisson ALgebras}

Throughout the paper $K$ denotes a field of characteristic zero. A vector space $A$ is called a Poisson algebra provided that, beside addition, it has two $K$-bilinear operations which are related by derivation. First, with respect to multiplication, $A$ is a commutative associative algebra with unit; denote the multiplication by $a \cdot b$ (or $a b)$, where $a, b \in A$. Second, $A$ is a Lie algebra; traditionally here the Lie operation is denoted by the Poisson brackets $\{a, b\}$, where $a, b \in A$. It is also assumed that these two operations are connected by the Leibnitz rule

$$
\{a \cdot b, c\}=a \cdot\{b, c\}+b \cdot\{a, c\}, \quad a, b, c \in A .
$$

Poisson algebras arise naturally in different areas of algebra and topology. Let us give two examples. First, consider the commutative and associative polynomial ring $K[X, Y]$. It is turned into a Poisson algebra if we introduce the Poisson bracket as follows:

$$
\{f, g\}=\frac{\partial f}{\partial X} \frac{\partial g}{\partial Y}-\frac{\partial f}{\partial Y} \frac{\partial g}{\partial X}, \quad f, g \in K[X, Y] .
$$

This is the Hamiltonian algebra $\mathbf{H}_{2}$; see Section 5

Second, given a Lie algebra $L$, there is a standard way of constructing a corresponding Poisson algebra $P=P(L)$ as follows. As a vector space $P$ coincides with $S(L)$, the symmetric algebra of $L$. Note that $S(L)$ is identified with the commutative polynomial ring $K\left[v_{1}, v_{2}, \ldots\right]$, where $v_{1}, v_{2}, \ldots$ is a linear basis of $L$ over the field $K$. Now define the Poisson brackets $\{$,$\} on P$ as follows: $\left\{v_{i}, v_{j}\right\}=\left[v_{i}, v_{j}\right]$ in $L$, and extend by linearity and by derivations to all of $P$. For example, $\left\{v_{i} \cdot v_{j}, v_{k}\right\}=v_{i} \cdot\left\{v_{j}, v_{k}\right\}+v_{j} \cdot\left\{v_{i}, v_{k}\right\}$, etc. In this way we construct the free Poisson algebra on the set $X$; see Section 2. We remark that $P=P(L)$ can also be defined via the universal enveloping algebra $U(L)$; see [5].

A Poisson algebra $A$ is PI (i.e. satisfies a polynomial identity) if there exists a non zero polynomial in the free Poisson algebra which vanishes under any substitution

Received by the editors August 23, 2004 and, in revised form, February 22, 2005.

2000 Mathematics Subject Classification. Primary 17B63, 17B01, 16P90, 16R10.

This research was partially supported by Grant RFBR-04-01-00739. 
in $A$. Note that many Poisson algebras are PI. For example, any Poisson algebra which is finitely generated as an associative algebra is PI. The goal of this paper is to study such PI algebras. Similar to the associative and to the Lie case, our main tools here are combinatorial, mainly applying the representations of the symmetric group $S_{n}$. We therefore assume that the characteristic of the base field $K$ is zero. Also, some special type of polynomials, customary, play a major role here; see below.

Some of the main features of the combinatorial PI theory in the associative case are:

P1. The exponential bound on the codimensions, and the integrality of that exponent.

P2. The cocharacters lie in a hook.

Note that these properties no longer hold in the Lie PI case. The main results of this paper are that the Poisson PI algebras do satisfy these properties P1 and P2, as well as their consequences.

Let $x_{1}, x_{2}, \ldots$ be associative and noncommutative variables. The polynomial

$$
s_{n}=s_{n}\left(x_{1}, \ldots, x_{n}\right)=\sum_{\sigma \in S_{n}}(-1)^{\sigma} x_{\sigma(1)} \cdots x_{\sigma(n)}
$$

is called the standard polynomial (of degree $n$ ), where $(-1)^{\sigma}$ is the sign of $\sigma$. It plays an important role in the theory of associative PI algebras. For example, the celebrated Amitsur-Levitsky Theorem says that $M_{k}(R)$, the $k \times k$ matrices over a commutative ring $R$, satisfies the identity $s_{2 k} \equiv 0$. Furthermore, by a well-known Amitsur's theorem, any associative PI algebra satisfies some $\ell$ 's power of some $k$-th standard polynomial $\left(s_{k}\right)^{\ell} \equiv 0$.

In the theory of Poisson PI algebras, the analogue of the standard polynomial is

$$
\sum_{\sigma \in S_{2 n}}(-1)^{\sigma}\left\{x_{\sigma(1)}, x_{\sigma(2)}\right\} \cdots\left\{x_{\sigma(2 n-1)}, x_{\sigma(2 n)}\right\},
$$

called a standard customary polynomial, where $\{$,$\} are the Poisson brackets.$ The products $\left\{x_{i_{1}}, x_{i_{2}}\right\} \cdots\left\{x_{i_{2 n-1}}, x_{i_{2 n}}\right\}$ are called customary monomials, and their linear combinations - customary polynomial. These polynomials where introduced by D. Farkas [8, 9 .

In this paper we study in detail the customary identities satisfied by a Poisson PI algebra. This is done by studying the intersection of the ideal of the identities of a given Poisson PI algebra - with the (free) space of the multilinear customary polynomials in $2 n$ variables. In the free case, this space of multilinear customary polynomials of degree $2 n$ has an over-exponential growth, more or less like $n$ ! Our main result asserts that when we mod-out the identities, the growth of the customary codimensions of a Poisson PI algebra is exponential - and with an integer exponent (Theorem 8.1). The exponential bound is a "Poisson" analogue of the corresponding result for associative PI algebras [17. The integrality of the exponential growth is a "Poisson" analogue of the corresponding result of A. Giambruno and M. Zaicev on the integrality of the exponent for associative algebras [10. In Section 9 we apply Theorem 8.1 to prove that the tensor product of PI Poisson algebras is again a PI-algebra.

In Section 5 we study in detail the customary identities of the Poisson algebras $\mathbf{H}_{2 k}$. As Lie algebras, these algebras are one-dimensional central extensions of the 
simple infinite dimensional Lie algebras of the Hamiltonian series $\mathrm{H}_{2 k}$. We refer to $\mathbf{H}_{2 k}$ as Hamiltonian Poisson algebras as well.

In Section 6 we construct the Grassmann-Poisson algebra $\mathbf{G}$ and study in detail all its polynomial identities. This algebra $\mathbf{G}$ is an analogue of the associative Grassmann algebra.

The authors are grateful to A.Guterman for attracting their attention to this subject.

\section{Customary polynomials}

The main objective of this section is the study of the customary polynomial identities. These polynomials and their importance in the Poisson PI theory are discussed below.

Recall from Section 1 the construction of the Poisson algebra $P=P(L)$ of a Lie algebra $L$. In the special case when $L=L(X)$ is the free Lie algebra on the set of variables $X$, this yields $P(L(X))=F(X)$, the free Poisson algebra on the variables $X$ [18. Let us describe its basis. We consider the Hall basis family $R(X)=\bigcup_{n=1}^{\infty} R_{n}(X)$ of $L(X)$, where the elements of $R_{n}$ are Lie elements of degree $n$ in $X$ [2]. In particular, $R_{1}=\left\{x_{i} \mid i \in I\right\}, R_{2}=\left\{\left[x_{i}, x_{j}\right] \mid i<j, i, j \in I\right\}$. These basis elements are well-ordered, and the order is compatible with the degree. For a detailed description of the $R_{j}$ 's see [2].

Let $R(X)=\left\{v_{j} \mid j=1,2, \ldots\right\}$. For simplicity, let the $v_{j}$ 's also denote the respective elements in $S(L)$, then $F(X)=S(L)=K\left[v_{j} \mid j=1,2, \ldots\right]$.

Consider the free Poisson algebra $F(X)$ with the countable generating set $X=$ $\left\{x_{i} \mid i \in \mathbb{N}\right\}$. Let $P_{n}=P_{n}(X) \subset F(X)$ be the subspace of all the multilinear elements of degree $n$ in $\left\{X_{1}, \ldots, X_{n}\right\}$. By the PBW-theorem, the following spanning set is a basis:

$$
P_{n}=\left\langle\prod_{i=1}^{n} X_{i}^{\alpha_{i}} \cdot \prod_{1 \leq i<j \leq n}\left\{X_{i}, X_{j}\right\}^{\beta_{i j}} . \prod_{v \in R_{s}\left(X_{1}, \ldots, X_{n}\right) ; s \geq 3} v^{\gamma_{v}}\right\rangle_{K},
$$

where all $X_{i}, i=1, \ldots, n$, appear exactly once, so in particular $\alpha_{i}, \beta_{i j}, \gamma_{v} \in\{0,1\}$. Also consider the subspace $Q_{2 n} \subseteq P_{2 n}$ spanned by the elements

$$
\left\{x_{\alpha_{1}}, x_{\alpha_{2}}\right\} \cdot\left\{x_{\alpha_{3}}, x_{\alpha_{4}}\right\} \cdots\left\{x_{\alpha_{2 n-1}}, x_{\alpha_{2 n}}\right\} .
$$

Following D. Farkas [8, we call the elements of $Q_{2 n}$ customary polynomials. The following spanning set is a canonical basis for $Q_{2 n}$ :

$$
\begin{gathered}
Q_{2 n}=\left\langle\left\{x_{\tau(1)}, x_{\tau(2)}\right\} \cdot\left\{x_{\tau(3)}, x_{\tau(4)}\right\}\right. \\
\tau(1)<\tau(2), \tau(3)<\tau(4), \ldots, \tau(2 n-1)<\tau(2 n), \\
\tau(1)<\tau(3)<\cdots<\tau(2 n-1)\rangle_{K} .
\end{gathered}
$$

Denote by $T_{2 n}$ the set of the permutations of $S_{2 n}$ that satisfy the above conditions. Note that $\left|T_{2 n}\right|$ is equal to the number of partitions of the set $\{1,2, \ldots, 2 n\}$ into pairs; this number will be denoted by $h(2 n)=\left|T_{2 n}\right|$. It is well known that

$$
\left|T_{2 n}\right|=\frac{(2 n) !}{2^{n} \cdot n !}=1 \cdot 3 \cdot 5 \cdots(2 n-1) .
$$

Moreover, $T_{2 n}$ can be chosen as a transversal of the left cosets of the wreath-product $Z_{2} \succ S_{n}$ in $S_{2 n}$. 
In addition, we define $R_{n} \subseteq P_{n}$ to be the subspace spanned by products of customary monomials and singletons:

$$
\left\{x_{\alpha_{1}}, x_{\alpha_{2}}\right\} \cdot\left\{x_{\alpha_{3}}, x_{\alpha_{4}}\right\} \cdots\left\{x_{\alpha_{2 m-1}}, x_{\alpha_{2 m}}\right\} \cdot x_{\alpha_{2 m+1}} \cdots x_{\alpha_{n}} .
$$

We call such elements extended customary polynomials. The following spanning set is a basis of $R_{n}$ :

$$
\begin{aligned}
& R_{n}=\left\langle\left\{x_{\tau(1)}, x_{\tau(2)}\right\} \cdot\left\{x_{\tau(3)}, x_{\tau(4)}\right\}\right. \cdots\left\{x_{\tau(2 m-1)}, x_{\tau(2 m)}\right\} \cdot x_{\tau(2 m+1)} \cdots x_{\tau(n)} \mid \\
& \tau \in S_{n}, \quad 0 \leq 2 m \leq n, \\
& \tau(1)<\tau(2), \tau(3)<\tau(4), \ldots, \tau(2 m-1)<\tau(2 m), \\
& \tau(1)<\tau(3)<\cdots<\tau(2 m-1), \\
&\tau(2 m+1)<\tau(2 m+2)<\cdots<\tau(n)\rangle_{K} .
\end{aligned}
$$

Note that the inclusion $R_{n} \subset P_{n}$ is strict; for example, $\left\{\left\{x_{1}, x_{2}\right\}, x_{3}\right\}$ belongs to $P_{3}$ but not to $R_{3}$.

The definition of a Poisson PI algebra is standard, the identities being elements of the free Poisson algebra $F(X)$ of countable rank. The collection of Poisson algebras satisfying the same set of identities is called a variety. We assume that the basic facts about varieties of PI algebras are known to the reader; see e.g. [7] and $[2]$.

The importance of customary polynomials is explained by the following fact, which was discovered by D. Farkas.

Theorem 2.1 ([8]). Suppose that $\mathcal{V}$ is a nontrivial variety of Poisson algebras. Then it satisfies a nontrivial customary identity:

$$
\sum_{\tau \in T_{2 n}} \alpha_{\tau}\left\{x_{\tau(1)}, x_{\tau(2)}\right\} \cdot\left\{x_{\tau(3)}, x_{\tau(4)}\right\} \cdots\left\{x_{\tau(2 n-1)}, x_{\tau(2 n)}\right\} \equiv 0 .
$$

\section{Codimensions}

Denote $X=\left\{x_{i} \mid i \in \mathbb{N}\right\}$. Let $\mathcal{V}$ be a variety of Poisson algebras, the polynomial identities being some elements of $F(X)$. Denote by $F(\mathcal{V}, X)$ the relatively free algebra of $\mathcal{V}$ generated by $X$. This induces the natural epimorphism $\pi: F(X) \rightarrow$ $F(\mathcal{V}, X)$. Denote by $Q_{2 n}(\mathcal{V}, X), R_{n}(\mathcal{V}, X)$, and $P_{n}(\mathcal{V}, X)$ the natural images of $Q_{2 n}, R_{n}$, and $P_{n}$, respectively. In what follows, the standard generating set $X$ may be omitted and we simply write, for example, $Q_{2 n}(\mathcal{V})$, etc. This yields the following three sequences of codimensions:

- $q_{2 m}(\mathcal{V})=\operatorname{dim} Q_{2 m}(\mathcal{V}) \quad$ (customary codimensions),

- $r_{n}(\mathcal{V})=\operatorname{dim} R_{n}(\mathcal{V}) \quad$ (extended customary codimensions),

- $p_{n}(\mathcal{V})=\operatorname{dim} P_{n}(\mathcal{V}) \quad$ (regular codimensions).

Define the upper and lower exponents for these sequences as follows:

$$
\begin{aligned}
& \overline{\operatorname{Exp}}^{Q} \mathcal{V}=\limsup _{n \rightarrow \infty} \sqrt[2 n]{q_{2 n}(\mathcal{V})}, \quad \underline{\operatorname{Exp}}^{Q} \mathcal{V}=\liminf _{n \rightarrow \infty} \sqrt[2 n]{q_{2 n}(\mathcal{V})}, \\
& \overline{\operatorname{Exp}}^{R} \mathcal{V}=\limsup _{n \rightarrow \infty} \sqrt[n]{r_{n}(\mathcal{V})}, \quad \underline{\operatorname{Exp}}^{R} \mathcal{V}=\liminf _{n \rightarrow \infty} \sqrt[n]{r_{n}(\mathcal{V})} \\
& \overline{\operatorname{Exp}} \mathcal{V}=\limsup _{n \rightarrow \infty} \sqrt[n]{p_{n}(\mathcal{V})}, \quad \quad \underline{\operatorname{Exp}} \mathcal{V}=\liminf _{n \rightarrow \infty} \sqrt[n]{p_{n}(\mathcal{V})}
\end{aligned}
$$

These limits always exist (of course they might be infinite). If the upper and the lower limits coincide, we use the notations $\operatorname{Exp}^{Q} \mathcal{V}, \operatorname{Exp}^{R} \mathcal{V}$, and $\operatorname{Exp} \mathcal{V}$, the 
corresponding exponents of the variety $\mathcal{V}$. By an exponent of a Poisson algebra $A$ denote the corresponding exponent of the variety $\mathcal{V}=\operatorname{var}(A)$ generated by this algebra.

Lemma 3.1. Let $\mathcal{V}$ be a variety of Poisson algebras. Then the sequences $q_{2 m}(\mathcal{V})$ and $r_{n}(\mathcal{V})$ are related by the following formula:

$$
r_{n}(\mathcal{V})=\sum_{0 \leq 2 m \leq n}\left(\begin{array}{c}
n \\
2 m
\end{array}\right) \cdot q_{2 m}(\mathcal{V}), \quad n \in \mathbb{N} .
$$

Proof. Fix $X_{n}=\left\{x_{1}, \ldots, x_{n}\right\}$. Let $2 m \leq n$, and let $Y=Y_{2 m} \subseteq X_{n}$ be a subset of cardinality $\left|Y_{2 m}\right|=2 m$. Denote by $\left\{v_{2 m, \alpha}(Y) \mid 1 \leq \alpha \leq q_{2 m}(\mathcal{V})\right\}$ a basis of $Q_{2 m}(\mathcal{V}, Y)$. Also, if $X \backslash Y=\left\{x_{i_{1}}, \ldots, x_{i_{n-2 m}}\right\}$, then denote $Z_{Y}=x_{i_{1}} \cdots x_{i_{n-2 m}}$. We prove that the elements in the set

(3) $\left\{v_{2 m, \alpha}(Y) \cdot Z_{Y}|Y \subseteq X| Y \mid,=2 m, 0 \leq 2 m \leq n, 1 \leq \alpha \leq q_{2 m}(\mathcal{V})\right\}$

form a basis of $R_{n}\left(\mathcal{V}, X_{n}\right)$.

It is obvious that these elements $\operatorname{span} R_{n}\left(\mathcal{V}, X_{n}\right)$, so we prove they are linearly independent. Assume, by way of contradiction, that

$$
\sum_{2 m, \alpha, Y} \beta_{2 m, \alpha, Y}\left(v_{2 m, \alpha}(Y) \cdot Z_{Y}\right)=0
$$

and that some $\beta_{2 m, \alpha, Y} \neq 0$. Let $m_{0}$ be minimal with $\beta_{2 m_{0}, \alpha, \bar{Y}} \neq 0$, for some $\bar{Y}=Y_{2 m_{0}}$. Substituting $x_{i} \rightarrow 1$ in (4) for all the variables in $X_{n} \backslash \bar{Y}$, we obtain

$$
\sum_{\alpha} \beta_{2 m_{0}, \alpha, \bar{Y}} v_{2 m_{0}, \alpha}(\bar{Y})=0
$$

a contradiction.

Next, define the following two exponential generating functions:

$$
\begin{aligned}
& \mathcal{C}^{Q}(\mathcal{V}, z)=\sum_{m=0}^{\infty} \frac{q_{2 m}(\mathcal{V})}{(2 m) !} z^{2 m} ; \\
& \mathcal{C}^{R}(\mathcal{V}, z)=\sum_{n=0}^{\infty} \frac{r_{n}(\mathcal{V})}{n !} z^{n} .
\end{aligned}
$$

Simple manipulations with these series imply:

Corollary 3.1. $\mathcal{C}^{R}(\mathcal{V}, z)=\mathcal{C}^{Q}(\mathcal{V}, z) \cdot e^{z}$.

The following formula is the "Poisson analogue" of the relations between the proper and the ordinary codimensions of associative PI algebras [3], [7, and [15].

Corollary 3.2. Assume that $\operatorname{Exp}^{Q}(\mathcal{V})$ exists. Then $\operatorname{Exp}^{R}(\mathcal{V})=\operatorname{Exp}^{Q}(\mathcal{V})+1$.

Proof. Let $\operatorname{Exp}^{Q}(\mathcal{V})=\ell$. Then by Lemma 3.1 it is sufficient to show that $\sum_{0 \leq 2 m \leq n}\left(\begin{array}{c}n \\ 2 m\end{array}\right) \ell^{2 m}$ behaves asymptotically like $\frac{1}{2}(\ell+1)^{n}$. This clearly follows since $\ell>0$ and

$$
\sum_{0 \leq 2 m \leq n}\left(\begin{array}{c}
n \\
2 m
\end{array}\right) \cdot \ell^{2 m}=\frac{1}{2} \cdot\left((1-\ell)^{n}+(1+\ell)^{n}\right) .
$$




\section{Cocharacters And the aCtion of the Symmetric group}

Following D. Farkas [8], 9], we define the customary standard polynomial as follows. Let $n=2 m$. Recall from Section 2 the definition of the set $T_{2 n} \subseteq S_{2 n}$. Then the customary standard polynomial is

$$
\mathrm{St}_{2 m}=\mathrm{St}_{2 m}\left(x_{1}, \ldots, x_{2 m}\right)=\sum_{\tau \in T_{2 m}}(-1)^{\tau}\left\{x_{\tau(1)}, x_{\tau(2)}\right\} \cdots\left\{x_{\tau(2 m-1)}, x_{\tau(2 m)}\right\} .
$$

Clearly, $\operatorname{St}_{2 m}\left(x_{1}, \ldots, x_{2 m}\right)$ is customary. Recall the notation $h(2 m)=\left|T_{2 m}\right|=$ $\frac{(2 m) !}{m ! 2^{m}}$. The "full" standard polynomial is defined as

$$
\widetilde{\mathrm{St}}_{2 m}\left(x_{1}, \ldots, x_{2 m}\right)=\sum_{\pi \in S_{2 m}}(-1)^{\pi}\left\{x_{\pi(1)}, x_{\pi(2)}\right\} \cdots\left\{x_{\pi(2 m-1)}, x_{\pi(2 m)}\right\} .
$$

It is easy to see that

$$
\widetilde{\mathrm{St}}_{2 m}\left(x_{1}, \ldots, x_{2 m}\right)=\frac{(2 m) !}{h(2 m)} \operatorname{St}_{2 m}\left(x_{1}, \ldots, x_{2 m}\right)=2^{m} m ! \operatorname{St}_{2 m}\left(x_{1}, \ldots, x_{2 m}\right) .
$$

Lemma 4.1. Let $n=2 m$ be even. Then

$$
\begin{aligned}
\mathrm{St}_{2 m+2}\left(x_{1}, \ldots, x_{2 m+2}\right) & =\mathrm{St}_{2 m}\left(x_{1}, \ldots, x_{2 m}\right) \cdot\left\{x_{2 m+1}, x_{2 m+2}\right\} \\
& -\sum_{j=1}^{2 m} \mathrm{St}_{2 m}\left(x_{1}, \ldots, x_{j-1}, x_{2 m+1}, x_{j+1}, \ldots, x_{2 m}\right) \cdot\left\{x_{j}, x_{2 m+2}\right\} .
\end{aligned}
$$

Proof. Here is a hint of the proof. Note that the number of customary monomials

$$
\left\{x_{\pi(1)}, x_{\pi(2)}\right\} \cdots\left\{x_{\pi(2 m+1)}, x_{\pi(2 m+2)}\right\}
$$

on both sides is the same - with no cancellations on either side. The minus signs on the right are caused by switching $x_{j}$ and $x_{2 m+1}$.

By definition, $\mathrm{St}_{2}=\left\{x_{1}, x_{2}\right\}$. An example of the previous lemma is

$$
\mathrm{St}_{4}=\left\{x_{1}, x_{2}\right\} \cdot\left\{x_{3}, x_{4}\right\}-\left\{x_{3}, x_{2}\right\} \cdot\left\{x_{1}, x_{4}\right\}-\left\{x_{1}, x_{3}\right\} \cdot\left\{x_{2}, x_{4}\right\} .
$$

In the odd case $n=2 m+1$, the customary standard polynomial is an "extended" polynomial, defined as

$$
\mathrm{St}_{2 m+1}=\operatorname{St}_{2 m+1}\left(x_{1}, \ldots, x_{2 m+1}\right)=\sum_{j=1}^{2 m+1}(-1)^{j+1} \operatorname{St}_{2 m}\left(x_{1}, \ldots, \hat{x}_{j}, \ldots, x_{2 m+1}\right) \cdot x_{j} .
$$

The "full" customary standard polynomial is

$$
\begin{aligned}
\widetilde{\mathrm{St}}_{2 m+1}\left(x_{1}, \ldots, x_{2 m+1}\right)= & \sum_{\pi \in S_{2 m+1}}(-1)^{\pi}\left\{x_{\pi(1)}, x_{\pi(2)}\right\} \cdots\left\{x_{\pi(2 m-1)}, x_{\pi(2 m)}\right\} \\
& \cdot x_{\pi(2 m+1)} \\
= & 2^{m} m ! \mathrm{St}_{2 m+1}\left(x_{1}, \ldots, x_{2 m+1}\right) .
\end{aligned}
$$

Cocharacters. Let $\sigma \in S_{n}$. Then the mapping $\sigma\left(x_{i}\right)=x_{\sigma(i)}$ is extended to an automorphism of the free Poisson algebra. The spaces $P_{n}(\mathcal{V})$ and $R_{n}(\mathcal{V})$ are $S_{n^{-}}$ modules, and similarly $Q_{2 m}(\mathcal{V})$ is an $S_{2 m}$-module. This defines the corresponding sequences of cocharacters $\chi_{n}^{P}(\mathcal{V}), \chi_{n}^{R}(\mathcal{V})$, and $\chi_{2 m}^{Q}(\mathcal{V})$, which are characters of the corresponding symmetric groups. 
Let $\chi_{n}$ be an $S_{n}$ character and let $\lambda \vdash n$ be a partition (or a Young diagram). As usual, we say that $\lambda$ appears in $\chi_{n}$ if the corresponding irreducible character $\chi_{\lambda}$ (also denoted $\chi^{\lambda}$ in the literature) appears in $\chi_{n}$ with a nonzero multiplicity.

Let $\lambda \vdash n$ and consider the largest subdiagram $\mu \subset \lambda$ with even columns (i.e. with columns of even length). Then $\mu$ can be calculated as follows. Let $\lambda^{\prime}$ be the conjugate partition of $\lambda$, and write $\lambda^{\prime}=\left(2 n_{1}+\epsilon_{1}, \ldots, 2 n_{s}+\epsilon_{s}, 1^{t}\right)$ where $\epsilon_{i} \in\{0,1\}$ and $n_{s}>0$. Then $\mu^{\prime}=\left(2 n_{1}, \ldots, 2 n_{s}\right)$. Clearly, $\mu \vdash 2 m$ where $m=n_{1}+\cdots+n_{s}$. Denote by $T=T_{\lambda}$ the standard Young tableau constructed as follows. Fill in the numbers $1,2, \ldots, 2 m$ consecutively into the first column of $\mu$, into the second column of $\mu$, etc., then insert the numbers $2 m+1, \ldots, n$ into the boxes corresponding to $\epsilon_{1}, \ldots, \epsilon_{s}$; finally, fill in the remaining boxes in the first row. For example let $\lambda=(5,3,2)$. Then $T_{\lambda}$ is

\begin{tabular}{|l|l|l|l|l|}
\hline 1 & 3 & 5 & 9 & 10 \\
\cline { 1 - 2 } 2 & 4 & 6 & \multicolumn{2}{|c}{} \\
\cline { 1 - 2 } 7 & 8 & \multicolumn{3}{|c}{} \\
\cline { 1 - 2 } & &
\end{tabular}

As usual, given $\lambda \vdash n$ and a tableau $T$ of shape $\lambda$, let $e_{T}=R_{T} C_{T}$ denote the corresponding semi-idempotent in $K S_{n}$. Here $R_{T}$ is the sum of the row permutations of $T$, while $C_{T}$ is the signed sum of the column permutations of $T$.

Return now to the above specific tableau $T_{\lambda}$, and define the following corresponding multilinear element of the free Poisson algebra:

$$
\tilde{f}_{\lambda}=R_{T} C_{T}\left(\left\{x_{1}, x_{2}\right\} \cdots\left\{x_{2 m-1}, x_{2 m}\right\} \cdot x_{2 m+1} \cdots x_{n}\right) .
$$

Next, define the polynomial

$$
f_{\lambda}=\mathrm{St}_{\lambda_{1}^{\prime}} \cdots \mathrm{St}_{\lambda_{s+t}^{\prime}}
$$

where $s+t=\lambda_{1}$ is the length of the first row of the diagram $\lambda$ and $s$ is the number of columns of length $\geq 2$. Note that $f_{\lambda}$ is not multilinear, but up to a nonzero scalar it is obtained from $\tilde{f}_{\lambda}$ as follows: substitute one variable for the variables of the first row of $T_{\lambda}$, substitute a second variable for the variables of the second row, etc. Let us show that $\tilde{f}_{\lambda}$ is a nonzero element of the free Poisson algebra. We use the Hamiltonian algebras (see their definition in Section 5 below). Let $X_{1}, \ldots, X_{m}, Y_{1}, \ldots, Y_{m} \in \mathbf{H}_{2 m}$, substitute $x_{1}=X_{1}, x_{2}=Y_{1}, \ldots, x_{2 m-1}=X_{m}$, $x_{2 m}=Y_{m}$, and finally substitute 1 for the rest of the variables. Since $\left\{X_{i}, Y_{j}\right\}=$ $\delta_{i, j}$, it follows that the result of this substitution in $f_{\lambda}$ is equal to 1 .

Also define $g_{\lambda}=C_{T} \tilde{f}_{\lambda}$. Clearly, $g_{\lambda}$ is skew-symmetric with respect to the sets of variables whose indices are written in each column of $\lambda$. It is known that $R_{T} C_{T} R_{T} C_{T}=\gamma R_{T} C_{T}$ where $\gamma \neq 0$, hence $R_{T} \cdot g_{\lambda} \neq 0$, so conclude that $g_{\lambda} \neq 0$.

We say that $\lambda$ lies in the hook $H(i, j)$ if $\lambda_{i+1} \leq j$. We say that this hook has leg of width $j$ and arm of width $i$. We call $H(i, 0)$ a horizontal strip.

Lemma 4.2. Let $A$ be a Poisson algebra which, as an associative algebra, is generated by $k$ elements. Then the customary and the extended customary cocharacters lie in a horizontal strip:

$$
\begin{aligned}
& \chi_{2 n}^{Q}(A)=\sum_{\lambda \vdash 2 n ; \lambda_{i}^{\prime} \text { even; } \lambda_{1}^{\prime} \leq k} m_{\lambda} \chi_{\lambda} ; \\
& \chi_{n}^{R}(A)=\sum_{\lambda \vdash n ; \lambda_{1}^{\prime} \leq k+1} \bar{m}_{\lambda} \chi_{\lambda} .
\end{aligned}
$$


Proof. Let $A$ be generated by $a_{1}, \ldots, a_{k}$. Consider first the customary cocharacter $\chi_{2 n}^{Q}(A)$. Suppose $m_{\lambda} \neq 0$ for some $\lambda$ with $\lambda_{1}^{\prime}>k$. Consider a simple submodule (of $K S_{n}$ ) that corresponds to $\lambda$. We may assume that such a submodule is generated by an element $f$ that corresponds to $C_{T_{\lambda}} R_{T_{\lambda}} C_{T_{\lambda}}$ for some tableau $T_{\lambda}$. Such an element has a set of $\ell$ alternating variables, with $\ell=\lambda_{1}^{\prime}>k$.

Let $f$ be alternating, say, on the first $\ell$ variables. Then clearly, for any $a_{i_{1}}, a_{i_{2}}, \ldots$ $\in\left\{a_{1}, \ldots, a_{k}\right\}, f\left(a_{i_{1}}, a_{i_{2}}, \ldots\right)=0$ (there must be a repetition among $a_{i_{1}}, a_{i_{2}}, \ldots, a_{\ell}$ as $\ell>k$ ). Since $f$ is multilinear, we can restrict ourselves to substitutions $x_{i} \rightarrow \bar{x}_{i}$ which are products of the generators $a_{1}, \ldots, a_{k}$. Now, customary polynomials are derivations in each variable: $f(a b, \ldots)=a f(b, \ldots)+b f(a, \ldots)$, etc. It follows that with such substitutions,

$$
f\left(\bar{x}_{1}, \bar{x}_{2}, \ldots\right)=\sum_{(i)} w_{(i)} \cdot f\left(a_{i_{1}}, a_{i_{2}}, \ldots\right)=0,
$$

where $w_{(i)}$ are some monomials in the $a_{j}^{\prime}$ s. This completes the proof for $\chi_{2 n}^{Q}(A)$.

Consider now $\chi_{n}^{R}(A)$. A set of at most $k$ skew-symmetric letters in brackets can be extended by at most one letter in singletons because they commute. Now apply the above same arguments.

\section{Hamiltonian ALgebras}

An important role in the theory of infinite dimensional Lie algebras is played by the (infinite dimensional) simple Lie algebras. Elie Cartan introduced four series of such algebras. These are: the general series $\mathrm{W}_{n}$ (Witt algebras); the special series $\mathrm{S}_{n}$ (not to be confused with the symmetric group!); the Hamiltonian series $\mathrm{H}_{2 n}$; and the contact series $\mathrm{K}_{2 n+1}$, where $n \in \mathbb{N}$. V. Kac proved that these algebras together with the Kac-Moody algebras and the Virasoro algebra, are the only infinite dimensional graded simple Lie algebras of polynomial growth (as algebras) over an algebraically closed field of characteristic zero [11. Actually, he imposed some technical assumptions that were later removed by O. Mathieu [13.

The algebras $\mathbf{H}_{2 m}$ play an important role in later sections, and hence are described below. For more detailed discussions see [11, 13. The derivations of the commutative polynomial ring $B_{n}=K\left[X_{1}, \ldots, X_{n}\right]$ form a Lie algebra with respect to the commutator. This Lie algebra $\mathrm{W}_{n}=\operatorname{Der} B_{n}$ is called the Witt algebra. Any element $a \in \mathrm{W}_{n}$ is of the form $a=\sum_{i=1}^{n} f_{i} \frac{\partial}{\partial X_{i}}$, where $f_{i} \in B_{n}$. The Hamiltonian Lie algebra $\mathrm{H}_{2 m}$ is defined as the subalgebra of $\mathrm{W}_{2 m}$, spanned as follows:

$$
\mathrm{H}_{2 m}=\left\langle\sum_{i=1}^{m}\left(-\frac{\partial f}{\partial X_{m+i}} \frac{\partial}{\partial X_{i}}+\frac{\partial f}{\partial X_{i}} \frac{\partial}{\partial X_{m+i}}\right) \mid f \in K\left[X_{1}, \ldots, X_{2 m}\right]\right\rangle_{K} .
$$

Consider the polynomial ring $\mathbf{H}_{2 m}=K\left[X_{1}, \ldots, X_{m}, Y_{1}, \ldots, Y_{m}\right]$, set $\left\{X_{i}, Y_{j}\right\}=$ $\delta_{i, j}$ and extend this bracket by the derivation rule. The result is a Poisson algebra with respect to the natural multiplication, where the Poisson brackets are computed as follows:

$$
\{f, g\}=\sum_{i=1}^{m} \frac{\partial f}{\partial X_{i}} \frac{\partial g}{\partial Y_{i}}-\frac{\partial f}{\partial Y_{i}} \frac{\partial g}{\partial X_{i}}, \quad f, g \in \mathbf{H}_{2 m}
$$


Identify the variables $Y_{1}, \ldots, Y_{m}$ with $X_{m+1}, \ldots, X_{2 m}$, then one checks that the following mapping $\Phi$ is an epimorphism of Lie algebras: $\mathbf{H}_{2 m} \rightarrow \mathrm{H}_{2 m}$

$$
\Phi: f \mapsto \sum_{i=1}^{m}\left(-\frac{\partial f}{\partial X_{m+i}} \frac{\partial}{\partial X_{i}}+\frac{\partial f}{\partial X_{i}} \frac{\partial}{\partial X_{m+i}}\right), \quad f \in \mathbf{H}_{2 m} .
$$

This homomorphism has a one-dimensional kernel, consisting of the constant polynomials. Thus, as a Lie algebra, $\mathbf{H}_{2 m}$ is a one-dimensional central extension of the simple Hamiltonian Lie algebra $\mathrm{H}_{2 m}$. We shall refer also to the Poisson algebra $\mathbf{H}_{2 m}$ as the Hamiltonian algebra.

The following theorem describes the module structure of the customary and of the extended customary polynomials for the free Poisson algebra. Similar methods were used by V. Drensky to study varieties of representations of Lie algebras which are nilpotent of class 2 [6]. The structure here is similar: all the simple $S_{n}$-modules appear, and with multiplicity one.

Theorem 5.1. Let $F=F(X), X=\left\{x_{i} \mid i \in \mathbb{N}\right\}$, be the free Poisson algebra. Then

(1) $\chi_{n}^{R}(F)=\sum_{\lambda \vdash n} \chi_{\lambda}$, for $n \in \mathbb{N}$;

(2) $\chi_{2 n}^{Q}(F)=\sum_{\lambda \vdash 2 n ; \lambda_{i}^{\prime} \text { even }} \chi_{\lambda}$, for $n \in \mathbb{N}$.

Proof. Fix the following notations: $R_{n}=R_{n}(F), Q_{2 n}=Q_{2 n}(F), r_{n}=\operatorname{dim} R_{n}$, and $q_{2 n}=\operatorname{dim} Q_{2 n}$; see Section [3. Recall that $R_{n}(F)$ has the canonical basis given by (2). Let $g(n)$ denote the number of involutions in $S_{n}$. An element in (2) corresponds to the involution (in cycle notation)

$$
(\tau(1), \tau(2)) \cdots(\tau(2 m-1), \tau(2 m)),
$$

and the assumptions on the orders in (2) imply that this correspondence is a bijection. Thus $r_{n}=g(n)$. On the other hand, it is well-known that

$$
g(n)=\sum_{\lambda \vdash n} \operatorname{dim} \chi_{\lambda}
$$

(see e.g. [21, proof of Proposition 2]; it also follows from the identity on Schur functions [12, Problem 1.5.4]).

We next show that every partition $\lambda \vdash n$ does appear in the module $R_{n}$. Fix $\lambda \vdash n$ and let $\lambda^{\prime}=\left(m_{1}, \ldots, m_{k}\right)$ be its conjugate, where $k=\lambda_{1}$. Recall that

$$
f_{\lambda}=\mathrm{St}_{m_{1}} \cdot \mathrm{St}_{m_{2}} \cdots \mathrm{St}_{m_{k}} \text {. }
$$

We need to show that $f_{\lambda} \neq 0$ in the free Poisson algebra, which is not that obvious. We prove it by showing that $f_{\lambda}\left(X_{1}, Y_{1}, \ldots\right) \neq 0$ for a certain substitution in $\mathbf{H}_{2 s}$, the Hamiltonian algebra, where $2 s \geq m_{1}$. The following element $B(m) \in \mathbf{H}_{2 s}$ has the following nonzero value, since $\left\{X_{i}, Y_{j}\right\}=\delta_{i, j}$ :

$$
B(m)= \begin{cases}\operatorname{St}_{m}\left(X_{1}, Y_{1}, \ldots, X_{k}, Y_{k}\right)=1, & m=2 k, \\ \operatorname{St}_{m}\left(X_{1}, Y_{1}, \ldots, X_{k}, Y_{k}, X_{k+1}\right)=X_{k+1}, & m=2 k+1 .\end{cases}
$$

Thus

$$
f_{\lambda}\left(X_{1}, Y_{1}, \ldots\right)=B\left(m_{1}\right) B\left(m_{2}\right) \cdots B\left(m_{k}\right) \neq 0 .
$$

Hence $R_{n}$ contains a simple submodule corresponding to $\lambda$. Applying (5), conclude that for all $\lambda \vdash n, R_{n}$ contains a corresponding simple module $V_{\lambda}$, and with multiplicity one. This proves (1). 
To prove the second claim, recall that $Q_{2 n} \subset R_{2 n}$. The same computations (6) and (17) also prove that all $\lambda \vdash 2 n$ with even (length) columns appear in $Q_{2 n}$. Since $Q_{2 n} \subset R_{2 n}$, these appear with multiplicity 1 . If $\lambda \vdash 2 n$ has a column of odd length, then the monomials in $f_{\lambda}$ contain at least one singleton, hence $f_{\lambda} \notin Q_{2 n}$. This proves the second claim.

The above theorem clearly implies

Corollary 5.1. Let $\mathcal{V}$ be a variety of PI Poisson algebras; then

(1) $\chi_{n}^{R}(\mathcal{V})=\sum_{\lambda \vdash n} m_{\lambda} \chi_{\lambda}$, for $n \in \mathbb{N}$, and all $m_{\lambda} \in\{0,1\}$. Moreover, let

$$
f_{\lambda}=\mathrm{St}_{m_{1}} \cdot \mathrm{St}_{m_{2}} \cdots \mathrm{St}_{m_{k}}
$$

then $m_{\lambda}=0$ if and only if $f_{\lambda}$ is an identity of $\mathcal{V}$.

(2) $\chi_{2 n}^{Q}(\mathcal{V})=\sum_{\lambda \vdash 2 n ; \lambda_{i}^{\prime} \text { even }} \bar{m}_{\lambda} \chi_{\lambda}$, for $n \in \mathbb{N}$, and all $\bar{m}_{\lambda} \in\{0,1\}$. Moreover, there is a similar statement about $f_{\lambda}=\mathrm{St}_{m_{1}} \cdot \mathrm{St}_{m_{2}} \cdots \mathrm{St}_{m_{k}}$, where the $m_{i}$ 's are even.

Theorem [5.1 has some additional implications. From formula (1) it follows that $q_{2 n}$ is equal to the number of all the partitions of $\{1,2, \ldots, 2 n\}$ into pairs, i.e. $q_{2 n}=h(2 n)$ in the previous notations. We obtain the well-known fact (e.g. [12, Problem 1.5.5 or 7.(2.4)]):

$$
h(2 n)=\sum_{\lambda \vdash 2 n, \lambda_{i}^{\prime} \text { even }} \operatorname{dim} \chi_{\lambda} .
$$

Corollary 5.2. Consider the Hamiltonian algebra $\mathbf{H}_{2 k}$. Then

(1) $\chi_{2 n}^{Q}\left(\mathbf{H}_{2 k}\right)=\sum_{\lambda \vdash 2 n, \lambda_{1}^{\prime} \leq 2 k, \lambda_{i}^{\prime} \text { even }} \chi_{\lambda}$;

(2) $\chi_{n}^{R}\left(\mathbf{H}_{2 k}\right)=\sum_{\lambda \vdash n, \lambda_{1}^{\prime} \leq 2 k+1} \chi_{\lambda}$;

(3) $\operatorname{Exp}^{Q}\left(\mathbf{H}_{2 k}\right)=2 k$;

(4) $\operatorname{Exp}^{R}\left(\mathbf{H}_{2 k}\right)=2 k+1$.

Proof. It is well known that claim (1) implies claim (3) and that claim (2) implies claim (4). We therefore prove claims (1), (2) only. By Lemma 4.2, all the diagrams for $Q_{2 n}\left(\mathbf{H}_{2 k}\right)$ are in the horizontal strip of height $2 k$, and all the diagrams of $R_{n}\left(\mathbf{H}_{2 k}\right)$ are in the horizontal strip of height $2 k+1$. On the other hand, the above computations show that the respective diagrams are present in $Q_{2 n}\left(\mathbf{H}_{2 k}\right)$ and $R_{n}\left(\mathbf{H}_{2 k}\right)$ (for the biggest columns in $R_{n}\left(\mathbf{H}_{2 k}\right)$, of length $2 k+1$, we change the last substitution in (6) into $x_{2 k+1}=1$ ).

Corollary 5.3. Consider the Hamiltonian algebra $\mathbf{H}_{2}$. Then

(1) $q_{2 n}\left(\mathbf{H}_{2}\right)=\frac{1}{n+1}\left(\begin{array}{c}2 n \\ n\end{array}\right)$ is the $n$-th Catalan number;

(2) $r_{n}\left(\mathbf{H}_{2}\right)$ is the Motzkin number.

Proof. By the previous corollary, $Q_{2 n}\left(\mathbf{H}_{2}\right)$ consists of one diagram $\lambda=(n, n)$ and by the hook formula $\operatorname{dim} \chi_{\lambda}=\frac{(2 n) !}{n !(n+1) !}=\frac{1}{n+1}\left(\begin{array}{c}2 n \\ n\end{array}\right)$. For the second claim see e.g. [19, Problem 7.16.b]. 


\section{The Poisson-Grassmann algebra}

Let $\Lambda$ denote the (ordinary) associative Grassmann algebra, generated by the countable-dimensional vector space $V=\left\langle e_{1}, e_{2}, \ldots\right\rangle_{K}$. The multiplication in this algebra is denoted by $\wedge$, i.e. let $a, b \in \Lambda$; then denote the product by $a \wedge b$. To make this algebra commutative, define a new multiplication - as follows: let $a, b \in \Lambda$; then

$$
a \cdot b=\frac{1}{2}(a \wedge b+b \wedge a) .
$$

This new multiplication is clearly bilinear, and $1 \in \Lambda$ remains a unit with respect to this multiplication. The associativity $a \cdot(b \cdot c)=(a \cdot b) \cdot c$ is easily verified. (Note: given any noncommutative algebra, one can try to "make" it commutative by introducing a similar new - multiplication. Then that - is associative if and only if the algebra satisfies the identity $[a,[b, c]]=0$.)

Define also the Poisson brackets on $\Lambda$ as $\{a, b\}=[a, b]=a \wedge b-b \wedge a$; then it is easy to verify the Leibnitz rule: $\{a \cdot b, c\}=a \cdot\{b, c\}+b \cdot\{a, c\}$. Thus $\Lambda$ is a Poisson algebra with respect to these two new operations. We call it the PoissonGrassmann algebra and denote it by $\mathbf{G}$.

Assume $K$ is of characteristic zero. It is well known that all the associative polynomial identities of $\Lambda$ follow from the identity $[[x, y], z] \equiv 0$. Note that $\{x, y\}$ is central in $\Lambda$, hence $\{x, y\} \cdot z=[x, y] \wedge z$. It follows that in evaluating the polynomial $\operatorname{St}_{n}\left(x_{1}, \ldots, x_{n}\right)$ in $\mathbf{G}$, we can essentially evaluate it in $\Lambda$. Thus for example,

$$
\begin{aligned}
&(-1)^{\pi}\left\{e_{\pi(1)}, e_{\pi(2)}\right\} \cdots\left\{e_{\pi(2 m-1)}, e_{\pi(2 m)}\right\} \\
&=(-1)^{\pi}\left[e_{\pi(1)}, e_{\pi(2)}\right] \wedge \cdots \wedge\left[e_{\pi(2 m-1)}, e_{\pi(2 m)}\right] \\
&=(-1)^{\pi} 2^{m} e_{\pi(1)} \wedge e_{\pi(2)} \wedge \cdots \wedge e_{\pi(2 m-1)} \wedge e_{\pi(2 m)} \\
&=2^{m} e_{1} \wedge e_{2} \wedge \cdots \wedge e_{2 m} .
\end{aligned}
$$

Note also that $\left\{1+e_{1}, e_{2}\right\}=\left\{e_{1}, e_{2}\right\}$, hence

$$
\mathrm{St}_{2 m}\left(1+e_{1}, e_{2}, \ldots, e_{2 m}\right)=\mathrm{St}_{2 m}\left(e_{1}, e_{2}, \ldots, e_{2 m}\right) .
$$

This and similar calculations yield that for any $k$ and $r$

$$
\begin{aligned}
\mathrm{St}_{2 m}\left(1+e_{1}, e_{2}, \ldots, e_{2 m}\right) \cdot\left(1+e_{1}\right)^{r} & =2^{m}\left|T_{2 m}\right| e_{1} \wedge \cdots \wedge e_{2 m} \\
\mathrm{St}_{2 m+1}\left(1+e_{1}, e_{2}, \ldots, e_{2 m+1}\right) \cdot\left(1+e_{1}\right)^{r} & =\alpha e_{1} \wedge \cdots \wedge e_{2 m+1} \\
& +2^{m}\left|T_{2 m}\right| e_{2} \wedge \cdots \wedge e_{2 m+1} .
\end{aligned}
$$

Theorem 6.1. Let $\mathbf{G}$ be the Poisson-Grassmann algebra. Then

(1) $\chi_{2 n}^{Q}(\mathbf{G})=\chi_{\left(1^{2 n}\right)}$ and $q_{2 n}(\mathbf{G})=1, n \in \mathbb{N}$;

(2) $\chi_{n}^{R}(\mathbf{G})=\sum_{r=1}^{n} \chi_{\left(r, 1^{n-r}\right)}$ and $p_{n}(\mathbf{G})=r_{n}(\mathbf{G})=2^{n-1}, n \in \mathbb{N}$;

(3) $\operatorname{Exp}^{Q} \mathbf{G}=1$;

(4) $\operatorname{Exp}^{R} \mathbf{G}=\operatorname{Exp} \mathbf{G}=2$;

(5) all the identities of $\mathbf{G}$ follow from $\{\{x, y\}, z\} \equiv 0$.

Proof. Since $\mathbf{G}$ satisfies the identity $\{\{x, y\}, z\} \equiv 0$, it follows that $P_{n}(\mathbf{G})=R_{n}(\mathbf{G})$. We show next that all the partitions in the hook $H(1,1)$ appear in $R_{n}(\mathbf{G})$. Let $\lambda=$ $\left(r, 1^{n-r}\right) \vdash n$; then $f_{\left(r, 1^{n-r}\right)}=\mathrm{St}_{n-r+1} \cdot x_{1}^{r-1}$, and $f_{\left(r, 1^{n-r}\right)}\left(1+e_{1}, e_{2}, \ldots, e_{n-r+1}\right) \neq$ 
0 by (8) and (9). Therefore

$$
p_{n}(\mathbf{G})=r_{n}(\mathbf{G}) \geq \sum_{r=1}^{n} \operatorname{dim} \chi_{\left(r, 1^{n-r}\right)}=\sum_{r=1}^{n}\left(\begin{array}{c}
n-1 \\
r-1
\end{array}\right)=2^{n-1} .
$$

Let $\mathcal{U}$ be the variety of Poisson algebras defined by the identical relation $\{\{x, y\}$, $z\} \equiv 0$. Consider its consequence obtained as a result of the "customarization" process [8]:

$$
\begin{aligned}
0 & \equiv\left\{\left\{x_{1} \cdot x_{2}, y\right\}, z\right\}-x_{1} \cdot\left\{\left\{x_{2}, y\right\}, z\right\}-\left\{\left\{x_{1}, y\right\}, z\right\} \cdot x_{2} \\
& =\left\{x_{1}, y\right\} \cdot\left\{x_{2}, z\right\}+\left\{x_{1}, z\right\} \cdot\left\{x_{2}, y\right\}
\end{aligned}
$$

This identity allows one to change letters on the second places in $Q_{2 n}(\mathcal{U})$. Recall that we can also freely interchange brackets and letters inside brackets. Therefore, the space $Q_{2 n}(\mathcal{U})$ is spanned by one monomial. Hence $q_{2 n}(\mathbf{G}) \leq q_{2 n}(\mathcal{U}) \leq 1$ for all $n \geq 1$. By Lemma 3.1.

$$
r_{n}(\mathbf{G}) \leq r_{n}(\mathcal{U})=\sum_{0 \leq 2 m \leq n}\left(\begin{array}{c}
n \\
2 m
\end{array}\right) q_{2 m}(\mathcal{U}) \leq \sum_{0 \leq 2 m \leq n}\left(\begin{array}{c}
n \\
2 m
\end{array}\right)=2^{n-1}
$$

Now the arguments above, along with (10) and (11), yield claims (1)-(4).

To prove claim (5) recall that we obtained the upper bound (11) relying on the identity $\{\{x, y\}, z\} \equiv 0$ only. Therefore, all the identities of $\mathbf{G}$ follow from this single identity.

Theorem 6.2. Let $\mathcal{V}$ be a variety of Poisson algebras such that $\mathbf{G} \notin \mathcal{V}$. Then the customary characters $\chi_{n}^{R}(\mathcal{V})$ and $\chi_{2 n}^{Q}(\mathcal{V})$ lie in a horizontal strip.

Proof. By assumption $\mathbf{G} \notin \mathcal{V}$. Hence, there exists a diagram $\lambda=\left(r, 1^{n-r}\right)$ from the space $P_{n}(\mathbf{G})=R_{n}(\mathbf{G})$ such that the respective identity is satisfied in $\mathcal{V}$. Since the multiplicities of the extended customary polynomials in the free Poisson algebra are equal to one, we conclude that $\mathcal{V}$ satisfies $\mathrm{St}_{n-r+1} \cdot x_{1}^{r-1} \equiv 0$. Assume first that $n-r+1$ is odd; then the standard identity contains singletons. Substitute $x_{1}=1$ and conclude that $\mathrm{St}_{n-r} \equiv 0$. Assume next that $n-r+1$ is even. Consider the result of the following partial linearization: Substitute $x_{1}+y_{1}$ for $x_{1}$; then take the homogeneous component of degree $r-1$ in $y_{1}$. In this component we set $y_{1}=1$ and obtain the standard identity $\mathrm{St}_{n-r+1} \equiv 0$.

Let $2 m$ be the degree of the standard identity in both cases. By Corollary 5.1 this implies that all the diagrams of $R_{n}(\mathcal{V})$ lie in the horizontal strip of height $2 m+1$.

\section{VARIETIES OF CUSTOMARY ALMOST POLYNOMIAL GROWTH}

The goal of this section is to show the critical role played by $\mathbf{H}_{2}$ and $\mathbf{G}$ (Theorem 7.3) in studying the customary growth of Poisson algebras.

Let $\mathcal{V}$ be a variety of Poisson algebras and let $H$ be a Poisson algebra. We write $H \notin_{R} \mathcal{V}$ if there exists an extended customary identity of $\mathcal{V}$ which is not satisfied in $H$. We say that $\mathcal{V}$ is of almost polynomial growth if it has exponential growth of $p_{n}(\mathcal{V})$, but any proper subvariety $\mathcal{U} \subset \mathcal{V}$ has a polynomial growth of $p_{n}(\mathcal{U})$. We also write $\mathcal{U} \subset_{R} \mathcal{V}$ to denote that $\mathcal{U} \subset \mathcal{V}$, but these two varieties differ by some nontrivial extended customary identity. Similarly, we say that $\mathcal{V}$ is of customary 
almost polynomial growth if $r_{n}(\mathcal{V})$ is of exponential growth, but for any $\mathcal{U} \subset_{R} \mathcal{V}$ the sequence of $r_{n}(\mathcal{U})$ is of polynomial growth.

We now study the variety $\operatorname{var} \mathbf{H}_{2}$. In the proof of the next two theorems we use the following notation. Let $f\left(x_{1}, \ldots, x_{m}, y_{1}, \ldots\right)$ be a polynomial; then we use the bar on the variables $x_{1}, \ldots, x_{m}$ to denote the skew-symmetrization in these variables:

$$
f\left(\bar{x}_{1}, \ldots, \bar{x}_{m}, y_{1}, \ldots\right) \sum_{\sigma \in S_{m}}(-1)^{\sigma} f\left(x_{\sigma(1)}, \ldots, x_{\sigma(m)}, y_{1}, \ldots\right) .
$$

Theorem 7.1. Consider the variety $\mathcal{V}$ generated by $\mathbf{H}_{2}$. Then

(1) $\chi_{2 n}^{Q}\left(\mathbf{H}_{2}\right)=\chi_{(n, n)}$;

(2) $\chi_{n}^{R}\left(\mathbf{H}_{2}\right)=\sum_{\lambda \vdash n, \lambda_{1}^{\prime} \leq 3} \chi_{\lambda}$;

(3) $\operatorname{Exp}^{Q}\left(\mathbf{H}_{2}\right)=2$;

(4) $\operatorname{Exp}^{R}\left(\mathbf{H}_{2}\right)=3$;

(5) all the customary identities of $\mathbf{H}_{2}$ follow from the identity $\mathrm{St}_{4} \equiv 0$.

Proof. Claims (1)-(4) are partial cases of Corollary [5.2, so we prove claim (5).

Denote by $\mathcal{U}$ the variety defined by the identity $\mathrm{St}_{4} \equiv 0$. Consider the submodule corresponding to $\lambda$ that is contained in $R_{n}(\mathcal{U})$. We shall prove that $\lambda_{1}^{\prime} \leq 3$. Recall that $\mathcal{V}=\operatorname{var} \mathbf{H}_{2} \subseteq \mathcal{U}$, and we are going to prove that $R_{n}(\mathcal{U})$ and $R_{n}(\mathcal{V})$ have the same characters. This would imply that all the customary identities of $\mathbf{H}_{2}$ follow from $\mathrm{St}_{4} \equiv 0$.

Here is a technical proof of claim (5). Clearly,

$\mathrm{St}_{4}=\left\{x_{1}, x_{2}\right\} \cdot\left\{x_{3}, x_{4}\right\}+\left\{x_{2}, x_{3}\right\} \cdot\left\{x_{1}, x_{4}\right\}+\left\{x_{3}, x_{1}\right\} \cdot\left\{x_{2}, x_{4}\right\}=\frac{1}{2}\left\{\bar{x}_{1}, \bar{x}_{2}\right\} \cdot\left\{\bar{x}_{3}, x_{4}\right\}$.

Thus, three skew-symmetric (i.e. alternating) variables inside two brackets yield zero. Four skew-symmetric letters inside three brackets also yield zero because then, at least three of these skew-symmetric letters enter two brackets. Decompose the following element with respect to position of $x_{4}$ in it:

$$
\begin{aligned}
0 \equiv\left\{\bar{x}_{1}, x_{5}\right\} \cdot\left\{\bar{x}_{2}, x_{6}\right\} \cdot\left\{\bar{x}_{3}, \bar{x}_{4}\right\} & \\
=\left\{\bar{x}_{1}, x_{5}\right\} & \cdot\left\{\bar{x}_{2}, x_{6}\right\} \cdot\left\{\bar{x}_{3}, x_{4}\right\}-\left\{\bar{x}_{1}, x_{5}\right\} \cdot\left\{\bar{x}_{2}, x_{6}\right\} \cdot\left\{x_{4}, \bar{x}_{3}\right\} \\
& +\left\{\bar{x}_{1}, x_{5}\right\} \cdot\left\{x_{4}, x_{6}\right\} \cdot\left\{\bar{x}_{2}, \bar{x}_{3}\right\}-\left\{x_{4}, x_{5}\right\} \cdot\left\{\bar{x}_{1}, x_{6}\right\} \cdot\left\{\bar{x}_{2}, \bar{x}_{3}\right\} .
\end{aligned}
$$

On the right, the first two terms are equal, while the other two terms equal zero by the above remark. Therefore, $\mathcal{U}$ satisfies the following identity:

$$
\left\{y_{1}, \bar{x}_{1}\right\} \cdot\left\{y_{2}, \bar{x}_{2}\right\} \cdot\left\{y_{3}, \bar{x}_{3}\right\} \equiv 0 .
$$

Thus, any customary element with three skew-symmetric letters is equal to zero. Since singletons commute, an extended customary element with four skew-symmetric letters is also zero. Hence, the cocharacter of $Q_{2 n}(\mathcal{U})$ has at most two rows and $R_{n}(\mathcal{U})$ has at most three rows. The result now follows.

Corollary 7.1. Consider the two varieties of Poisson algebras: $\mathcal{U}$ which is defined by $\mathrm{St}_{4}$, and $\operatorname{var}\left(\mathbf{H}_{2}\right)$. They are different, but both satisfy the same customary identities.

Proof. Namely, let us prove that $\mathcal{U}$ has no "pure Lie" identical relations at all, by latter we mean identical relations given by Lie polynomials. 
Indeed, let us consider the free Poisson algebra $F=F(X)$. Let $k \geq 0$; denote by $F^{(k)}$ the ideal of $F$ generated by all elements $\prod_{i=1}^{k}\left\{a_{i}, b_{i}\right\}$, where $a_{i}, b_{i} \in F$. We observe that $F^{(k)}$ are verbal ideals, i.e. they are stable under all endomorphisms of $F$. Indeed, let $\phi: F \rightarrow F$ be an endomorphism. Consider free generating elements $x, y \in X$. Suppose that $\phi(x)=\alpha+f, \phi(y)=\beta+g$, where $\alpha, \beta \in K$ and $f, g$ are of degree at least one with respect to $X$. Then $\{\phi(x), \phi(y)\}=\{f, g\}$. Moreover, we observe that the number of brackets in monomials is not decreasing after $\phi$. Hence, $F^{(k)}$ are verbal ideals. All embeddings $F^{(k+1)} \subset F^{(k)}, k \geq 0$, are proper; see the construction of $F(X)$ in Section 2, Remark that $\mathrm{St}_{4} \in F^{(2)}$. By our observation, all consequences of $\mathrm{St}_{4}$ belong to $F^{(2)}$, hence $\mathcal{U}$ has no "pure Lie" identities at all, i.e. homogeneous identities given by elements from $F^{(1)} \backslash F^{(2)}$.

On the other hand, the Lie algebra $\mathbf{H}_{2}$ is a central extension of the Lie algebra $\mathrm{H}_{2} \subset \mathrm{W}_{2}$, where the Witt Lie algebra $\mathrm{W}_{2}$ is a PI Lie algebra with exponential growth of codimensions [2]. More precisely (see Chapter 6, Section 43 in [16]) each infinite dimensional simple Lie algebra from the four Cartan series (in particular the algebra $\mathrm{H}_{2}$ ) satisfies the standard Lie identity of some degree $m+1$

$$
\left.\left.\sum_{\pi \in S_{m}}(-1)^{\pi}\left[\ldots\left[x_{0}, x_{\pi(1)}\right], x_{\pi(2)}\right], \ldots\right], x_{\pi(m)}\right] \equiv 0 .
$$

It is clear that the Poisson algebra $\mathbf{H}_{2}$ satisfies the same "pure Lie" Poisson identity.

Finally, by Theorem $7.1,5, \mathcal{U}$ and $\operatorname{var}\left(\mathbf{H}_{2}\right)$ have the same customary identities.

Theorem 7.2. Let $\mathcal{V}$ be a variety of Poisson algebras such that $\mathbf{H}_{2} \notin_{R} \mathcal{V}$ (see the beginning of this section). Then $\mathcal{V}$ satisfies the customary identity $\left\{x_{1}, x_{2}\right\}^{m} \equiv 0$ for some $m$.

Proof. By Corollary 5.2, $R_{n}\left(\mathbf{H}_{2}\right)$ consists of all the diagrams with three rows, the multiplicities being one. Then $R_{n}(\mathcal{V})$ does not contain a certain diagram $\lambda$ of the form $\lambda^{\prime}=3^{p} 2^{q} 1^{r}$. By standard methods this implies the following identity of $\mathcal{V}$ that corresponds to $\lambda$ :

$$
\widetilde{\mathrm{St}}_{3}^{p} \cdot{\widetilde{\mathrm{St}_{2}}}_{2}^{q} \cdot{\widetilde{\mathrm{St}_{1}}}_{1}^{r}=\left(\left\{\bar{x}_{1}, \bar{x}_{2}\right\} \bar{x}_{3}\right)^{p} \cdot\left\{\overline{\bar{x}}_{1}, \overline{\bar{x}}_{2}\right\}^{q} \cdot x_{1}^{r} \equiv 0 .
$$

Here, $x_{1}, x_{2}$ and $x_{3}$ alternate in the first factor, and $x_{1}$ and $x_{2}$ alternate in the second factor. Since $\left\{x_{2}, x_{1}\right\}=-\left\{x_{1}, x_{2}\right\}$, the alternation in the second factor can be removed, namely, the variety $\mathcal{V}$ satisfies the identity

$$
\left(\left\{\bar{x}_{1}, \bar{x}_{2}\right\} \cdot \bar{x}_{3}\right)^{p} \cdot\left\{x_{1}, x_{2}\right\}^{q} \cdot x_{1}^{r} \equiv 0 .
$$

Substitute $x_{3}=1$. Since in a Poisson algebra $\{1, x\}=0$, it follows that $\mathcal{V}$ satisfies

$$
\left\{x_{1}, x_{2}\right\}^{p} \cdot\left\{x_{1}, x_{2}\right\}^{q} \cdot x_{1}^{r}=\left\{x_{1}, x_{2}\right\}^{t} \cdot x_{1}^{r} \equiv 0,
$$

where $t=p+q$. We show that $\mathcal{V}$ satisfies $\left\{x_{1}, x_{2}\right\}^{t} \cdot x_{1}^{r-1} \equiv 0$. Repeating that argument clearly completes the proof. Substitute $x_{1}+y_{1}$ for $x_{1}$ and take the homogeneous component of degree 1 in $y_{1}$. Since $K$ is infinite $(\operatorname{char}(K)=0)$, that component is also an identity of $\mathcal{V}$. Now,

$$
\left\{x_{1}+y_{1}, x_{2}\right\}^{t}=\left\{x_{1}, x_{2}\right\}^{t}+\left\{y_{1}, x_{2}\right\} \cdot f\left(x_{1}, x_{2}\right)+\text { higher terms in } y_{1},
$$

for some polynomial $f\left(x_{1}, x_{2}\right)$. Similarly

$$
\left(x_{1}+y_{1}\right)^{r}=x_{1}^{r}+r y_{1} \cdot x_{1}^{r-1}+\text { higher terms in } y_{1} .
$$


Thus, after the substitution $x_{1}+y_{1}$ for $x_{1}$, that component of degree 1 in $y_{1}$ in the above identity is

$$
r\left\{x_{1}, x_{2}\right\}^{t} \cdot y_{1} \cdot x_{1}^{r-1}+\left\{y_{1}, x_{2}\right\} \cdot f\left(x_{1}, x_{2}\right) \cdot x_{1}^{r} .
$$

Substitute $y_{1}=1$ to deduce that $\mathcal{V}$ satisfies $\left\{x_{1}, x_{2}\right\}^{t} \cdot x_{1}^{r-1} \equiv 0$. The proof is complete.

The following is the main result of this section.

Theorem 7.3. Let $\mathcal{V}$ be a variety of Poisson algebras such that $\mathbf{H}_{2} \notin_{R} \mathcal{V}, \mathbf{G} \notin \mathcal{V}$. Then

(1) $r_{n}(\mathcal{V})$ is of polynomial growth;

(2) there exists $N_{0}$ such that $q_{2 n}(\mathcal{V})=0$ for all $n \geq N_{0}$.

Proof. By Theorem 6.2, the character $\chi_{n}^{R}(\mathcal{V})$ lies in a horizontal strip of some height $k$. Also, by Theorem [7.2 we have the identity $\left\{x_{1}, x_{2}\right\}^{t} \equiv 0$ for some $t$. Consider a diagram $\lambda=\left(\lambda_{1}, \ldots, \lambda_{k}\right)$ that appears in $R_{n}(\mathcal{V})$, and show that there is a bound on $\lambda_{2}, \ldots, \lambda_{k}$. Since the multiplicities $R_{n}(\mathcal{V})$ are bounded by 1 , the hook-formula then implies a polynomial bound on $r_{n}(\mathcal{V})$. Also, the columns of the diagrams in $Q_{2 n}(\mathcal{V})$ are of even length, hence the sizes of the diagrams in $Q_{2 n}(\mathcal{V})$ are bounded.

Write $\lambda^{\prime}=k^{n_{k}}(k-1)^{n_{k-1}} \ldots 2^{n_{2}} 1^{n_{1}}$. The identity corresponding to $\lambda$ is of the form

$$
\mathrm{St}_{k}^{n_{k}} \cdot \mathrm{St}_{k-1}^{n_{k-1}} \cdots \mathrm{St}_{2}^{n_{2}} \cdot \mathrm{St}_{1}^{n_{1}} .
$$

Consider $i \in\{k, k-1, \ldots, 2\}$ and find a bound on $n_{i}$.

Case 1: $i=2 m$. Then

$$
\begin{aligned}
\left(\mathrm{St}_{2 m}\right)^{n_{i}}=\left(\mathrm{St}_{2 m}\right. & \left.\left(x_{1}, \ldots, x_{2 m}\right)\right)^{n_{i}} \\
& =\left(\sum_{\tau \in T_{2 m}}(-1)^{\tau}\left\{x_{\tau(1)}, x_{\tau(2)}\right\} \cdots\left\{x_{\tau(2 m-1)}, x_{\tau(2 m)}\right\}\right)^{n_{i}} .
\end{aligned}
$$

The number of different pairs $\left\{x_{i_{1}}, x_{i_{2}}\right\}$ is $\left(\begin{array}{c}2 m \\ 2\end{array}\right)$. Assume $n_{i}>N=t\left(\begin{array}{c}2 m \\ 2\end{array}\right)$, where $t$ satisfies $\left\{x_{1}, x_{2}\right\}^{t} \equiv 0$.

Decompose (12) and consider one of the resulting monomials. Then at least one of the above pairs is raised to a sufficiently large power $\left\{x_{i_{1}}, x_{i_{2}}\right\}^{t^{\prime}}, t^{\prime} \geq t$. Hence, (12) is equal to zero.

Case 2: $i=2 m+1$. Here

$$
\left(\mathrm{St}_{2 m+1}\right)^{n_{i}}=\left(\sum_{j=1}^{2 m+1}(-1)^{j} \mathrm{St}_{2 m}\left(x_{1}, \ldots, \hat{x}_{j}, \ldots, x_{2 m+1}\right) \cdot x_{j}\right)^{n_{i}},
$$

where the hat denotes the omitted argument. The same arguments prove that this power is zero for sufficiently large $n_{i}$. The proof is complete.

This theorem easily yields the following two corollaries.

Corollary 7.2. (1) The variety generated by $\mathbf{G}$ is of almost polynomial growth.

(2) The variety generated by $\mathbf{H}_{2}$ is of customary almost polynomial growth. 
Proof. 1). By Theorem $6.1(2) \quad p_{n}(\mathbf{G})=2^{n-1}$, hence is exponential. By Theo$\operatorname{rem} \mathbf{7 . 1}(2), \quad \mathbf{H}_{2} \notin \operatorname{var}(\mathbf{G})$. If $\mathcal{V} \subset \operatorname{var}(\mathbf{G})$ is a proper subvariety, then $\mathbf{G} \notin \mathcal{V}$ as well as $\mathbf{H}_{2} \notin \mathcal{V}$, and by Theorem $7.3 p_{n}(\mathcal{V})=r_{n}(\mathcal{V})$ is polynomially bounded. (Recall that $P_{n}(\mathbf{G})=R_{n}(\mathbf{G})$; see Section 6 )

2). A similar proof, now applying Theorem 6.1(1), Theorem 7.1 (1) and Theorem 7.3

Corollary 7.3. Let $\mathcal{V}$ be a variety of customary almost polynomial growth. Then either the customary identities of $\mathcal{V}$ coincide with the customary identities of $\operatorname{var} \mathbf{G}$, or they coincide with those of $\operatorname{var} \mathbf{H}_{2}$.

Proof. Case 1. Assume that every extended customary identity of $\mathcal{V}$ is also an identity of $\mathbf{H}_{2}$; then $r_{n}\left(\operatorname{var} \mathbf{H}_{2}\right) \leq r_{n}(\mathcal{V})$ for all $n \in \mathbb{N}$. Recall that $\operatorname{var} \mathbf{H}_{2}$ is of exponential customary growth. By the assumption on $\mathcal{V}, r_{n}\left(\operatorname{var} \mathbf{H}_{2}\right)=r_{n}(\mathcal{V})$ hence $\mathcal{V}$ and $\operatorname{var} \mathbf{H}_{2}$ have the same extended customary identities, and we are done.

Case 2. Assume that every extended customary identity of $\mathcal{V}$ is also an identity of $\mathbf{G}$. By the same argument conclude that $\mathcal{V}$ and $\operatorname{var}(\mathbf{G})$ have the same extended customary identities.

Case 3. Assume neither Case 1 nor Case 2, namely $\mathbf{H}_{2} \notin_{R} \mathcal{V}$ and $\mathbf{G} \notin_{R} \mathcal{V}$. Then by Theorem $7.3 r_{n}(\mathcal{V})$ is of polynomial customary growth, contradicting the assumption that $r_{n}(\mathcal{V})$ is of exponential growth.

We say that a variety $\mathcal{V}$ has an intermediate customary growth if the sequence $r_{n}(\mathcal{V})$ lies properly between polynomial and exponential growth.

Corollary 7.4. There are no Poisson varieties of intermediate customary growth.

Proof. Suppose that $\mathcal{U}$ is a variety of intermediate customary growth. Since $\mathbf{H}_{2}$ and $\mathbf{G}$ have exponential customary growth, we have $\mathbf{H}_{2} \notin_{R} \mathcal{U}$ and $\mathbf{G} \notin_{R} \mathcal{U}$. By Theorem 7.3, $\mathcal{U}$ is of polynomial customary growth.

\section{EXPONENTIAL GROWTH}

Let $F=F(X), X=\left\{x_{i} \mid i \in \mathbb{N}\right\}$. In Section 2 we saw that $\operatorname{dim}\left(Q_{2 n}(F)\right)=$ $\left|T_{2 n}\right|=\frac{(2 n) !}{n ! 2^{n}}$. Then the Stirling formula implies that

$$
q_{2 n}(F)=\operatorname{dim} Q_{2 n}(F)=\left|T_{2 n}\right|=\frac{(2 n) !}{n ! 2^{n}} \approx \frac{n ! 2^{n}}{\sqrt{\pi n}}, \quad n \rightarrow \infty .
$$

Thus, the spaces $Q_{2 n}(F)$ of the customary polynomials have an over-exponential growth. We show that the customary growth is exponentially bounded if we impose a nontrivial identity. This is is the Poisson-analogue of the result which asserts that the codimension growth of a nontrivial variety of associative algebras is at most exponential [17. We use a technique similar to those in [21].

The following is the main result of the paper.

Theorem 8.1. Let $\mathcal{V}$ be a nontrivial variety of Poisson algebras. Then $\operatorname{Exp}^{Q} \mathcal{V}-$ the exponent of the customary growth - exists, and moreover, it is an integer.

The proof consists of several steps. Throughout this section we assume that $\mathcal{V}$ is a Poisson variety with a nontrivial identity. Let $n$ be even, let $\lambda \vdash n$ and assume $\lambda$ appears in $Q_{n}(\mathcal{V})$; then it appears in $Q_{n}(\mathcal{V})$ with multiplicity 1 . Denote 
$\lambda^{\prime}=\left(\alpha_{1}^{\beta_{1}}, \alpha_{2}^{\beta_{2}}, \ldots, \alpha_{s}^{\beta_{s}}\right)$, where $\alpha_{1}>\alpha_{2}>\cdots>\alpha_{s}>0$. We also assume that all $\alpha_{i}$ are even. The corresponding polynomial is

$$
f_{\lambda}=\mathrm{St}_{\alpha_{1}}^{\beta_{1}} \cdot \mathrm{St}_{\alpha_{2}}^{\beta_{2}} \cdots \mathrm{St}_{\alpha_{s}}^{\beta_{s}}
$$

By Corollary 5.1 we can assume that our variety satisfies $f_{\lambda} \equiv 0$. Let $\phi_{j}$ be the substitution of $z$ for $x_{j}$ and $\phi_{j}^{(i)}$ the substitution of $x_{i}$ for $x_{j}$, while other letters remain invariant.

For example, $\phi_{2}^{(4)}\left(\mathrm{St}_{2}\right)=\phi_{2}^{(4)}\left(\mathrm{St}_{2}\left(x_{1}, x_{2}\right)\right)=\mathrm{St}_{2}\left(x_{1}, x_{4}\right)$.

Consider some $j \in\left\{1, \ldots \alpha_{1}\right\}$; it corresponds to the $j$-th row in the diagram $\lambda$. Make the partial linearization of $f_{\lambda}$ with respect to $x_{j}$ by first substituting $x_{j} \rightarrow x_{j}+z$, and then taking the homogeneous part of degree one in $z$. Denote the result of this process by $f_{\lambda}^{(j)}$; it depends of course on (the row) $j$.

For the rows $j=1, \ldots, \alpha_{s}$ we have

$$
f_{\lambda}^{(j)}=f_{\lambda} \cdot \sum_{i=1}^{s} \beta_{i} \frac{\phi_{j}\left(\mathrm{St}_{\alpha_{i}}\right)}{\mathrm{St}_{\alpha_{i}}} .
$$

For the rows $j=\alpha_{s}+1, \ldots, \alpha_{s-1}$ we have

$$
f_{\lambda}^{(j)}=f_{\lambda} \cdot \sum_{i=1}^{s-1} \beta_{i} \frac{\phi_{j}\left(\mathrm{St}_{\alpha_{i}}\right)}{\mathrm{St}_{\alpha_{i}}}
$$

and so on. Finally, for the last rows $j=\alpha_{2}+1, \ldots, \alpha_{1}$ we obtain

$$
f_{\lambda}^{(j)}=f_{\lambda} \cdot \beta_{1} \frac{\phi_{j}\left(\mathrm{St}_{\alpha_{1}}\right)}{\mathrm{St}_{\alpha_{1}}} .
$$

We remark that the fractions above are introduced in order to simplify the notations.

Our goal is to prove that we can glue two boxes to $\lambda$ in some special corner; namely, if that extended diagram is $\mu$, then also $f_{\mu} \equiv 0$ is an identity of the algebra. Note that since $\alpha_{s-1}>\alpha_{s}$ and both are even, $\alpha_{s-1} \geq \alpha_{s}+2$.

Proposition 8.1. Let $\mathcal{V}$ satisfy $f_{\lambda} \equiv 0$; then $\mathcal{V}$ also satisfies $f_{\mu} \equiv 0$, where $\mu^{\prime}=$ $\left(\alpha_{1}^{\beta_{1}}, \ldots, \alpha_{s-1}^{\beta_{s-1}}, \alpha_{s}+2, \alpha_{s}^{\beta_{s}-1}\right)$.

Proof. Consider

$$
\beta_{s} f_{\lambda} \cdot\{z, t\}-\sum_{j=1}^{\alpha_{s}} f_{\lambda}^{(j)} \cdot\left\{x_{j}, t\right\}
$$

which is an identity of $\mathcal{V}$. Here we substitute $z=x_{\alpha_{s}+1}$ and $t=x_{\alpha_{s}+2}$. Note that $\phi_{j}^{\left(\alpha_{s}+1\right)}\left(\mathrm{St}_{\alpha_{k}}\right)=0$ for $k=1, \ldots, s-1$, since after the substitution the letter $x_{\alpha_{s}+1}$ appears twice inside the standard polynomial - which is alternating. Thus, the action of $\phi_{j}^{\left(\alpha_{s}+1\right)}$ can be restricted to the last factor $\mathrm{St}_{\alpha_{s}}^{\beta_{s}}$ only. In other words, when $z=x_{\alpha_{s}+1}$

$$
f_{\lambda}^{(j)} \cdot\left\{x_{j}, x_{\alpha_{s}+2}\right\}=\beta_{s} \mathrm{St}_{\alpha_{1}}^{\beta_{1}} \cdots \mathrm{St}_{\alpha_{s-1}}^{\beta_{s-1}} \cdot \mathrm{St}_{\alpha_{s}}^{\beta_{s}-1} \cdot\left(\phi_{j}^{\left(\alpha_{s}+1\right)}\left(\mathrm{St}_{\alpha_{s}}\right)\right) \cdot\left\{x_{j}, x_{\alpha_{s}+2}\right\} .
$$

By (14), this implies that $\mathcal{V}$ satisfies the following identity:

$\beta_{s} \mathrm{St}_{\alpha_{1}}^{\beta_{1}} \cdots \mathrm{St}_{\alpha_{s-1}}^{\beta_{s-1}} \cdot \mathrm{St}_{\alpha_{s}}^{\beta_{s}-1} \cdot\left(\mathrm{St}_{\alpha_{s}} \cdot\left\{x_{\alpha_{s}+1}, x_{\alpha_{s}+2}\right\}-\sum_{j=1}^{\alpha_{s}} \phi_{j}^{\left(\alpha_{s}+1\right)}\left(\mathrm{St}_{\alpha_{s}}\right) \cdot\left\{x_{j}, x_{\alpha_{s}+2}\right\}\right) \equiv 0$. 
By Lemma 4.1 the expression in the parentheses equals $\mathrm{St}_{\alpha_{s}+2}$. Thus, we obtained the desired identity $f_{\mu} \equiv 0$.

Assume the variety $\mathcal{V}$ satisfies the identity $f_{\lambda} \equiv 0$, where the last row of $\lambda$ is of length $q$ and the last column is of length $2 r$. The next proposition shows that we can glue to $\lambda$ an arbitrary "leg" of width $q$ and an arbitrary "arm" of width $2 r$, and the resulting diagram $\mu$ will yield the identity $f_{\mu} \equiv 0$ for the variety $\mathcal{V}$.

Proposition 8.2. Let $\lambda=\left(p^{2 r}, \lambda_{2 r+1}, \ldots, \lambda_{2(r+s)}, q^{2 l}\right)$ be a partition of $2 n$ with columns of even length, and

$$
\mu=\left(p+\alpha_{1}, \ldots, p+\alpha_{2 r}, \lambda_{2 r+1}, \ldots, \lambda_{2(r+s)}, q^{2 l}, \mu_{2(r+s+l)+1}, \mu_{2(r+s+l)+2}, \ldots\right)
$$

a partition of $2 N$, also with columns of even length. Then the customary identity $f_{\lambda} \equiv 0$ implies $f_{\mu} \equiv 0$.

Proof. As a first step we glue the leg, namely, denote

$$
\nu=\left(p^{2 r}, \lambda_{2 r+1}, \ldots, \lambda_{2(r+s)}, q^{2 l}, \mu_{2(r+s+l)+1}, \mu_{2(r+s+l)+2}, \ldots\right),
$$

a partition of $2 M$. We then show that the identity $f_{\nu} \equiv 0$ follows from the identity $f_{\lambda} \equiv 0$.

Let $T=T_{\lambda}$ be the standard Young tableau constructed as follows. Fill in the numbers $1,2, \ldots$ consecutively into the first column, into the second column, etc. As usual, for a tableau $T$ of shape $\lambda$, let $e_{T}=R_{T} C_{T}$ denote the corresponding semi-idempotent in $K S_{n}$. Since $R_{T} C_{T} R_{T} C_{T}=\gamma R_{T} C_{T}$, where $\gamma \neq 0$, conclude that $C_{T} R_{T} C_{T}$ is a nonzero element of $K S_{n}$. As in Section 4, define the following corresponding multilinear element of the free Poisson algebra:

$$
\tilde{f}_{\lambda}=R_{T} C_{T}\left(\left\{x_{1}, x_{2}\right\} \cdots\left\{x_{2 n-1}, x_{2 n}\right\}\right) .
$$

Also define $g_{\lambda}=C_{T} \tilde{f}_{\lambda}$. Clearly, this element is skew-symmetric with respect to the sets of variables whose indices are written in each column of $T_{\lambda}$. Note that $g_{\lambda} \in Q_{2 n}$, so $K S_{n} g_{\lambda} \subseteq Q_{2 n}$, namely $g_{\lambda}$ generates the irreducible module for $\lambda$ in $Q_{2 n}$. Consider $g_{\lambda}=g_{\lambda}\left(x_{1}, y_{1}, \ldots, x_{n}, y_{n}\right)$ (i.e. with a different set of variables).

Set $m=r+s+l$. Then $2 m$ is the length of the first $q$ columns of the diagram $\lambda$. By construction $g_{\lambda}$ is skew-symmetric in each of the next $q$ sets of variables $A_{i}=\left\{x_{(i-1) m+1}, y_{(i-1) m+1}, \ldots, x_{i m}, y_{i m}\right\}$, where $i=1, \ldots, q$. Moreover $g_{\lambda}$ is skewsymmetric in each of the sets of variables $A_{i}, i=q+1, \ldots, p$. Note that $\left|A_{i}\right|=\lambda_{i}^{\prime}$ is the length of the $i$-th columns, and each of the columns consists of the next variables.

Let $\tau \vdash 2(M-n)$ be the "leg"-partition which is being attached to $\lambda$, namely $\tau=\left(\mu_{2(r+s+l)+1}, \mu_{2(r+s+l)+2}, \ldots\right)$. Construct the multilinear element

$$
g_{\tau}=g_{\tau}\left(x_{n+1}, y_{n+1}, \ldots, x_{M-n}, y_{M-n}\right)
$$

in a similar way. Thus $g_{\tau}$ is skew-symmetric in each of the sets of variables $B_{i}=$ $\left\{x_{n+\tau_{1}^{\prime}+\cdots+\tau_{i-1}^{\prime}+1}, y_{n+\tau_{1}^{\prime}+\cdots+\tau_{i-1}^{\prime}+1}, \ldots, x_{n+\tau_{1}^{\prime}+\cdots+\tau_{i}^{\prime}}, y_{n+\tau_{1}^{\prime}+\cdots+\tau_{i}^{\prime}}\right\}, i=1, \ldots, q$. In particular, $B_{1}=\left\{x_{n+1}, y_{n+1}, \ldots, x_{n+\tau_{1}^{\prime}}, y_{n+\tau_{1}^{\prime}}^{\prime}\right\}$ and is not empty. Note that some $B_{j}$ might be empty.

Construct $g_{\lambda} \cdot g_{\tau}$, and in this product alternate each of the $q$ sets of variables $C_{i}=A_{i} \cup B_{i}, i=1, \ldots, q$. Note that this alternation corresponds to the first $q$ 
columns of $\nu$. Formally this is done as follows: Given the set $C_{i}=A_{i} \cup B_{i}$, let $S\left(C_{i}\right)$ be the symmetric group on this set. Denote $C_{\lambda, \mu}=S\left(C_{1}\right) \times \cdots \times S\left(C_{q}\right)$,

$$
C_{\lambda, \mu}^{-}=\sum_{\pi \in C_{\lambda, \mu}}(-1)^{\pi} \pi
$$

then consider $C_{\lambda, \mu}^{-}\left(g_{\lambda} \cdot g_{\tau}\right)$. Examine the decomposition into irreducibles of the left module generated by this element. First, the decomposition into irreducibles of the left module generated by $g_{\lambda} \cdot g_{\tau}$ is given by the Littlewood-Richardson $(\mathrm{L}-\mathrm{R})$ rule: the diagrams of these modules must contain $\lambda, \tau$. Let $\eta$ be such a diagram.

By $\mathrm{L}-\mathrm{R} \eta_{1}^{\prime} \leq\left|C_{1}\right|$; if $\eta_{1}^{\prime}=\left|C_{1}\right|$, then $\eta_{2}^{\prime} \leq\left|C_{2}\right|$; if $\eta_{1}^{\prime}=\left|C_{1}\right|$ and $\eta_{2}^{\prime}=\left|C_{2}\right|$, then $\eta_{3}^{\prime} \leq\left|C_{3}\right|$, etc. Because of the alternations of the variables of $C_{1}=A_{1} \cup B_{1}$ in $C_{\lambda, \mu}^{-}\left(g_{\lambda} \cdot g_{\tau}\right)$, the first column of $\eta$ must be of length at least $\left|C_{1}\right|$. Therefore $\eta_{1}^{\prime}=\left|C_{1}\right|$. A similar argument then shows that $\eta_{2}^{\prime}=\left|C_{2}\right|$, etc. Thus $\eta_{i}^{\prime}=\left|C_{i}\right|, \quad 1 \leq$ $i \leq q$. This implies that $\eta=\nu$, i.e. the left module generated by $C_{\lambda, \mu}^{-}\left(g_{\lambda} \cdot g_{\tau}\right)$ corresponds to the partition $\nu$. By applying $\mathbf{H}_{2 M}$ we now show that that module is nonzero in the free Poisson algebra.

Rewrite the element $g_{\lambda}$ as the sum of monomials (namely products of $\left\{x_{i}, x_{j}\right\}, i<$ $j,\left\{x_{i}, y_{j}\right\}$ or $\left.\left\{y_{i}, y_{j}\right\}, i<j\right)$. Check the coefficient of the monomial

$$
\begin{aligned}
\left\{x_{1}, y_{1}\right\} \cdots\left\{x_{m}, y_{m}\right\} \cdot \cdots \cdot\left\{x_{m(q-1)+1}, y_{m(q-1)+1}\right\} & \cdots\left\{x_{m q}, y_{m q}\right\} \\
\cdot & \left\{x_{m q+1}, y_{m q+1}\right\} \cdots\left\{x_{n}, y_{n}\right\} .
\end{aligned}
$$

Since we substitute $x_{i} \rightarrow X_{i}, y_{i} \rightarrow Y_{i}$ and $\left\{X_{i}, Y_{j}\right\}=\delta_{i, j}$ in $\mathbf{H}_{2 M}$, the result after that substitution will be zero for all the other monomials.

The structure of $g_{\lambda}$ implies that

$$
\left.g_{\lambda}=\alpha\left\{x_{1}, y_{1}\right\} \cdots\left\{x_{m}, y_{m}\right\} \cdots \cdot x_{m(q-1)+1}, y_{m(q-1)+1}\right\} \cdots\left\{x_{m q}, y_{m q}\right\} \cdot \tilde{g}_{\lambda}+h_{\lambda},
$$

where $\alpha \neq 0, \tilde{g}_{\lambda}=\left\{x_{m q+1}, y_{m q+1}\right\} \cdots\left\{x_{n}, y_{n}\right\}$, and the result of the above substitution in $h_{\lambda}$ is equal to 0 . The same arguments give us that

$$
\begin{aligned}
g_{\tau}= & \beta\left\{x_{n+1}, y_{n+1}\right\} \cdots\left\{x_{n+\tau_{1}^{\prime}}, y_{n+\tau_{1}^{\prime}}\right\} \cdots \\
& \cdot\left\{x_{n+\tau_{1}^{\prime}+\cdots+\tau_{q-1}^{\prime}+1}, y_{n+\tau_{1}^{\prime}+\cdots+\tau_{q-1}^{\prime}+1}\right\} \cdots\left\{x_{n+\tau_{1}^{\prime}+\cdots+\tau_{q}^{\prime}}, y_{n+\tau_{1}^{\prime}+\cdots+\tau_{q}^{\prime}}\right\}+h_{\tau}
\end{aligned}
$$

where $\beta \neq 0$ and the result of our substitution in $h_{\tau}$ is equal to 0 . Note that for this partition $\tau$, the element $g_{\tau}$ is without $\tilde{g}_{\tau}$ because the number of columns is less than or equal to $q$. Thus, the resulting element is

$$
\begin{gathered}
g_{\nu}=\gamma\left\{x_{1}, y_{1}\right\} \cdots\left\{x_{m}, y_{m}\right\} \cdot\left\{x_{n+1}, y_{n+1}\right\} \cdots\left\{x_{n+\tau_{1}^{\prime}}, y_{n+\tau_{1}^{\prime}}\right\} \cdots \\
\cdot\left\{x_{m(q-1)+1}, y_{m(q-1)+1}\right\} \cdots\left\{x_{m q}, y_{m q}\right\} \\
\cdot\left\{x_{n+\tau_{1}^{\prime}+\cdots+\tau_{q-1}^{\prime}+1}, y_{n+\tau_{1}^{\prime}+\cdots+\tau_{q-1}^{\prime}+1}\right\} \cdots\left\{x_{n+\tau_{1}^{\prime}+\cdots+\tau_{q}^{\prime}}, y_{n+\tau_{1}^{\prime}+\cdots+\tau_{q}^{\prime}}\right\} \cdot \tilde{g}_{\lambda}+h_{\nu},
\end{gathered}
$$

where $\gamma \neq 0$. So the result is nonzero and the element $g_{\nu}$ generates a nonzero irreducible module in $Q_{2 M}$ that corresponds to $\nu$. Since the multiplicities there are one, this element generates the same module as that of $f_{\nu}$. Clearly the identity $g_{\lambda} \equiv 0$ implies that $g_{\lambda} g_{\tau} \equiv 0$ hence also that $g_{\nu} \equiv 0$. Since $g_{\nu}$ and $f_{\nu}$ generate the same module, it follows that $f_{\nu} \equiv 0$. This shows that $f_{\nu} \equiv 0$ follows from $f_{\lambda} \equiv 0$.

Finally, we glue the arm $\tau$ to the diagram $\nu$. Say, the arm is $\tau=\left(\alpha_{1}, \ldots, \alpha_{2 r}\right)$. Note that $f_{\mu}=f_{\nu} \cdot f_{\tau}$ and conclude that $f_{\mu} \equiv 0$ follows from $f_{\lambda} \equiv 0$ as well.

Proposition 8.3. The cocharacters $\chi_{2 n}^{Q}(\mathcal{V})$ and $\chi_{n}^{R}(\mathcal{V})$ lie in a hook. 
Proof. Assume $\mathcal{V}$ satisfies a customary identity $f_{\lambda} \equiv 0$, where $\lambda$ has columns of even length. First, apply Proposition 8.1 successively to deduce that $\mathcal{V}$ satisfies $f_{\mu} \equiv 0$ where $\mu$ is any rectangle with even-length columns that contains $\lambda$. This is done as follows. Let $\lambda=\left(\alpha_{1}^{2 n_{1}}, \alpha_{2}^{2 n_{2}}, \ldots, \alpha_{k}^{2 n_{k}}\right)$ where $\alpha_{1}>\alpha_{2}>\cdots>\alpha_{k}>0$. Thus the $2 n_{1}+1$-th and the $2 n_{1}+2-$ th rows of $\lambda$ are of equal length $\alpha_{2}$, which is shorter than the $2 n_{1}-$ th row (whose length is $\alpha_{1}$ ). By Proposition 8.1 we can glue one box to the $2 n_{1}+1$-th row and one box to the $2 n_{1}+2$-th row of $\lambda$, and the resulted diagram still corresponds to an identity of $\mathcal{V}$. Now repeat this process until rows $2 n_{1}+1$ and $2 n_{1}+2$ also become of length $\alpha_{1}$. Then continue this process until obtaining a rectangle $\mu=\left(\alpha_{1}^{2 m}\right)$, where $m=n_{1}+\cdots+n_{k}$. By Proposition 8.1 $\mathcal{V}$ satisfies $f_{\mu} \equiv 0$. Furthermore, apply this process - of adding two vertical boxes - to this rectangle to obtain any rectangle $\mu$ of even height $\geq 2 m$ and length $\geq \alpha_{1}$, and $\mathcal{V}$ satisfies $f_{\mu} \equiv 0$.

Let $\eta$ be a diagram with even columns which contains $\lambda$. There is a maximal rectangle $\mu$ which is contained in $\eta$ and which contains $\lambda$ (there might be several such rectangles). Clearly, $\eta$ is obtained from such $\mu$ by gluing an arm and a leg. Thus, applying Proposition 8.2, deduce that $\mathcal{V}$ satisfies $f_{\eta} \equiv 0$. Together with Theorem 5.1 this implies that the cocharacters $\chi_{2 n}^{Q}(\mathcal{V})$ lie inside the hook $H\left(2 m, \alpha_{1}\right)$.

For the tail consisting of singletons we use commutativity and conclude that $\chi_{n}^{R}(\mathcal{V})$ can be obtained from $\chi_{2 m}^{Q}(\mathcal{V}), 2 m \leq n$, only by gluing a horizontal strip (in sense of [12]) to the respective $\lambda$ 's in $\chi_{2 m}^{Q}(\mathcal{V})$.

Proposition 8.4. $q_{2 n}(\mathcal{V})$, and $r_{n}(\mathcal{V})$ are exponentially bounded.

Proof. This follows from Proposition 8.3. Let $q_{2 n}^{R}(\mathcal{V})$ lie in the hook $H(i, j)$. The asymptotic calculations in Section 7 in [4] imply that $\overline{\operatorname{Exp}}^{Q} \mathcal{V} \leq i+j$. By Lemma 3.1 it follows that $\overline{\operatorname{Exp}}^{R} \mathcal{V} \leq i+j+1$.

Proposition 8.5. Let $k$ be an integer. Suppose that $\operatorname{Exp}^{Q} \mathcal{V}<k$; then $\overline{\operatorname{Exp}}^{Q} \mathcal{V} \leq$ $k-1$.

Proof. We discuss customary elements and sometimes omit the upper index $Q$ for convenience. Let $T$ be an integer and $\lambda \vdash n$; then define a new partition $\lambda^{T}$ as the intersection of $\lambda$ with the square $T \times T$. It follows from Proposition 8.2 that if $m_{\lambda^{2 T}}(\mathcal{V})=0$, then also $m_{\lambda}(\mathcal{V})=0$. By Proposition 8.3 all the diagrams of the character $\chi^{Q}(\mathcal{V})$ lie in the hook $H(s, s)$. Therefore if $t \geq s$, then $\lambda_{t} \leq s$, and similarly also $\lambda_{t}^{\prime} \leq s$, so that $\lambda_{t}+\lambda_{t}^{\prime} \leq 2 s$. Given $t \geq s$ and an even $n$, denote

$$
p_{n}(t)=\max _{\lambda \vdash n ; m_{\lambda} \neq 0}\left\{\lambda_{t}+\lambda_{t}^{\prime}\right\} ;
$$

then $p_{n}(t) \leq 2 s$. Define

$$
p(t)=\max _{n \text { even }} p_{n}(t) .
$$

Since $\lambda_{t} \geq \lambda_{t+1}$ and $\lambda_{t}^{\prime} \geq \lambda_{t+1}^{\prime}$, hence $p(t) \geq p(t+1)$. Therefore the following limit exists:

$$
p=\lim _{t \rightarrow \infty} p(t) .
$$

This means that there exists $t_{0}$ such that for any $t \geq t_{0}$ there exists $n_{t}$ and $\lambda \vdash n_{t}$ which appears in $Q_{n_{t}}(\mathcal{V})$, and $\lambda_{t}+\lambda_{t}^{\prime}=p$. Moreover let $n$ be arbitrary and let $\mu \vdash n$ be any partition appearing in $Q_{n}(\mathcal{V})$; then by definition, $\mu_{t_{0}}+\mu_{t_{0}}^{\prime} \leq p$. 
Therefore $\mu$ lies in the union of the $t_{0} \times t_{0}$ square and $H(i, j)$ - for some $i+j=p$. By the hook formula it follows that as $n$ tends to infinity, $\left(\operatorname{dim} \chi_{\mu}\right)^{\frac{1}{n}} \leq p$. Since the number of partitions of $n$ in $H(i, j)$ is polynomially bounded $\left(\leq n^{i+j}\right)$, it follows that $\overline{\operatorname{Exp}} \mathcal{V} \leq p$.

First, suppose that $p \leq k-1$; then it follows that $\overline{\operatorname{Exp}} \mathcal{V} \leq p \leq k-1$ and we are done.

Second, assume $p \geq k$ and derive a contradiction. By the very definition (17), there exist infinitely many values $t$ such that $p(t)=p$. Next, in (16), there exist infinitely many $n$ 's with $p_{n}(t)=p$. For each such $t$ and $n$ there exists $\lambda \vdash n$ appearing in $Q_{n}(\mathcal{V})$ such that $\lambda_{t}+\lambda_{t}^{\prime}=p$. Note that there are only finitely many pairs $(a, b)$ of nonnegative integers such that $a+b=p$. We conclude that there exist integers $i_{0}, j_{0} \geq 0$ such that $i_{0}+j_{0}=p$ and there exist infinitely many pairs $(\lambda, t)$ such that $\lambda_{t}=i_{0}$ and $\lambda_{t}^{\prime}=j_{0}$. Hence we obtain $p$ for infinitely many diagrams that lie in the union of the square $t_{0} \times t_{0}$ and the hook $H\left(i_{0}, j_{0}\right)$. We consider the intersections of these diagrams with the $t_{0} \times t_{0}$ square.

There are only finitely many diagrams contained in the $t_{0} \times t_{0}$ square, hence there exists a diagram $\mu$ which is the intersection of the $t_{0} \times t_{0}$ square with infinitely many such diagrams that yield the number $p$. Denote $|\mu|=n_{0}$, where $n_{0} \leq t_{0}^{2}$. Fix some $T \geq t_{0}$. We can find an irreducible character $\chi_{\lambda}$ appearing in $Q_{n}(\mathcal{V})$, and $t>T$, such that $\lambda_{T}=\lambda_{t}=i_{0}$ and $\lambda_{T}^{\prime}=\lambda_{t}^{\prime}=j_{0}$. Since $\lambda$ appears in $Q_{n}(\mathcal{V})$, by Proposition $8.2 m_{\lambda^{T}} \neq 0$. Consider the diagram $\lambda^{T}$ : except for its intersection with the $t_{0} \times t_{0}$ square it has a rectangular arm of width $i_{0}$, and a rectangular leg of a width $j_{0}$, both being of length $T$. The number of boxes in $\lambda^{T}$ is $n=n_{0}+\left(T-t_{0}\right) p$. By the hook formula

$$
\operatorname{dim} \lambda^{T} \approx C_{0} n^{\gamma} p^{n_{0}+\left(T-t_{0}\right) p}, \quad T \rightarrow \infty .
$$

This asymptotic is valid for infinitely many values of type $n=n_{0}+\left(T-t_{0}\right) p$, where $n_{0}, t_{0}$ and $p$ are fixed while $T$ is arbitrary such that $T \geq t_{0}$. These numbers form a subsequence of the arithmetic progression $n_{i}=m_{0}+i p, i=0,1,2, \ldots$. Since these numbers satisfy (18), we obtain

$$
\liminf _{i \rightarrow \infty}\left(q_{n_{i}}(\mathcal{V})\right)^{\frac{1}{n_{i}}} \geq p .
$$

Let us consider all the even numbers between such $n_{i}$ and $n_{i}-p$; say, we consider numbers of type $n_{i}-2$. From the structure of the customary polynomials (1) it follows that

$$
Q_{n}\left(\mathcal{V}, x_{1}, \ldots, x_{n}\right)=\sum_{j=2}^{n}\left\{x_{1}, x_{j}\right\} \cdot W_{j}\left(x_{2}, \ldots, \hat{x}_{j}, \ldots, x_{n}\right),
$$

where $W_{j}$ are some subspaces that are homomorphic images of $Q_{n-2}(\mathcal{V})$. This obviously implies that

$$
q_{n-2}(\mathcal{V}) \geq \frac{q_{n}(\mathcal{V})}{n-1}
$$

From (19) and (20) it follows that

$$
\liminf _{i \rightarrow \infty}\left(q_{n_{i}-2}(\mathcal{V})\right)^{\frac{1}{n_{i}-2}} \geq p .
$$

From this we conclude that $\operatorname{Exp} \mathcal{V} \geq p \geq k$, a contradiction to the assumption of the proposition. 
The proof of Theorem 8.1 now follows from Proposition 8.5

We now deduce a few corollaries from Theorem 8.1

Corollary 8.1. Let $A$ be a Poisson algebra with a nontrivial identity. Suppose that $A$ is finitely generated as an associative algebra. Then $\operatorname{Exp}^{Q} A$ is even and $\operatorname{Exp}^{R} A$ is odd.

Proof. Consider first $Q_{2 n}(A)$. By Lemma 4.2 the diagrams that appear in $Q_{2 n}(A)$ lie in a horizontal strip. In the proof of Theorem 8.1 all the diagrams are of even columns. In that proof it was shown that the cocharacters contain tableaux which - asymptotically - are rectangular. Hence $\operatorname{Exp}^{Q} A$ is even.

Now $\operatorname{Exp}^{R} A$ is odd by Corollary 3.2 .

From the proof of Theorem 8.1 also follows

Corollary 8.2. Let $\mathcal{V}$ be a nontrivial variety of Poisson algebras. Then the customary characters $\chi_{n}^{R}(\mathcal{V})$ and $\chi_{2 n}^{Q}(\mathcal{V})$ lie in a hook.

Corollary 8.3. Let $\mathcal{V}$ be a nontrivial variety of Poisson algebras. Then $\mathcal{V}$ satisfies a nontrivial customary identity of the special type

$$
\sum_{\pi \in S_{m}} \alpha_{\pi}\left\{x_{1}, y_{\pi(1)}\right\} \cdots\left\{x_{m}, y_{\pi(m)}\right\} \equiv 0, \quad \alpha_{\pi} \in K .
$$

Proof. The number of the above monomials $\left\{x_{1}, y_{\pi(1)}\right\} \cdots\left\{x_{m}, y_{\pi(m)}\right\}, \pi \in S_{m}$, is $m$ !, while the customary codimensions are exponentially bounded.

The following is an analogue of the well-known Amitsur's theorem.

Corollary 8.4. Let $\mathcal{V}$ be a nontrivial variety of Poisson algebras. Then $\mathcal{V}$ satisfies a power of a standard identity $\left(\mathrm{St}_{2 m}\right)^{k} \equiv 0$, for some integers $m, k$.

Proof. By Corollary 8.2 there exists a hook $H(i, j)$ such that all the characters of $\chi_{2 n}^{Q}(\mathcal{V})$ lie in this hook. Let $2 m>i$ and $k>j$; then the $2 m \times k$ rectangle $\lambda=\left(k^{2 m}\right)$ is not in $H(i, j)$, hence the corresponding customary polynomial $f_{\lambda}$ is an identity for $\mathcal{V}$. By Corollary 5.1, this identity is $f_{\lambda}=\left(\mathrm{St}_{2 m}\right)^{k} \equiv 0$.

\section{Tensor products of Poisson algebras}

Recall the notion of the tensor product of Poisson algebras (see e.g. 20]). Let $A$ and $B$ be two Poisson algebras. We consider the tensor product of the associative algebras $A \otimes_{K} B$ and equip it with the Poisson bracket via

$\left\{a_{1} \otimes b_{1}, a_{2} \otimes b_{2}\right\}=\left\{a_{1}, a_{2}\right\} \otimes b_{1} \cdot b_{2}+a_{1} \cdot a_{2} \otimes\left\{b_{1}, b_{2}\right\}, \quad a_{1}, a_{2} \in A, b_{1}, b_{2} \in B$.

It is easy to verify that $A \otimes B$ is a Poisson algebra. Moreover, with $1=1_{A} \in A$, $1 \otimes B$ and $B$ are isomorphic as Poisson algebras, so we can write $B \subseteq A \otimes B$ (here we use the fact that $\{1, a\}=0$ for all $a \in A$ ). It can be shown, for example, that

$$
\mathbf{H}_{2 m} \cong \underbrace{\mathbf{H}_{2} \otimes \cdots \otimes \mathbf{H}_{2}}_{m \text { times }} .
$$

The main result of this section is the following theorem. It is an analogue of the respective result of the third author for associative PI-algebras [17.

Theorem 9.1. Let $A$ and $B$ be Poisson PI algebras; then $A \otimes B$ is PI. 
The proof is given below. First, we construct extended customary monomials of special type. Denote $\underline{n}=\{1, \ldots, n\}$. We also denote $\underline{i}=\left\{i_{1}, \ldots, i_{k}\right\} \subseteq \underline{n}$, and $\underline{j}=$ $\underline{n} \backslash \underline{i}$ is its complement. Given such $\underline{i}=\left\{i_{1}, \ldots, i_{k}\right\} \subseteq \underline{n}$, with $\underline{j}=\underline{n} \backslash \underline{i}=\left\{j_{1}, \ldots, \bar{j}_{\ell}\right\}$, let $M_{\underline{i}}(x)=M_{\underline{i}}\left(x_{1}, \ldots, x_{2 n}\right)$ be the following extended customary monomial:

$$
M_{\underline{i}}(x)=\prod_{r=1}^{k}\left\{x_{2 i_{r}-1}, x_{2 i_{r}}\right\} \cdot \prod_{s=1}^{\ell}\left(x_{2 j_{s}-1} \cdot x_{2 j_{s}}\right)
$$

(Poisson brackets, then parenthesis) and let $M_{\underline{i}}^{c}(x)=M_{\underline{j}}(x)$ denote the corresponding "complementary" monomial:

$$
M_{\underline{i}}^{c}(x)=\prod_{r=1}^{k}\left(x_{2 i_{r}-1} \cdot x_{2 i_{r}}\right) \cdot \prod_{s=1}^{\ell}\left\{x_{2 j_{s}-1}, x_{2 j_{s}}\right\}
$$

(parenthesis, then Poisson brackets). Since each subset $\underline{i}=\left\{i_{1}, \ldots, i_{k}\right\} \subseteq \underline{n}$ determines such a monomial, in total there are $2^{n}$ such extended customary monomials $M_{\underline{i}}(x)$ in $x_{1}, \ldots, x_{2 n}$. The definition of the Poisson bracket in the tensor product yields, by induction, the following lemma.

Lemma 9.1. Let $A$ and $B$ be Poisson algebras and let $a_{1}, \ldots, a_{2 n} \in A, b_{1}, \ldots, b_{2 n} \in$ B. Then

$$
\begin{aligned}
\left\{a_{1} \otimes b_{1}, a_{2} \otimes b_{2}\right\} \cdots\left\{a_{2 n-1} \otimes b_{2 n-1},\right. & \left.a_{2 n} \otimes b_{2 n}\right\} \\
& =\sum_{\underline{i} \subseteq \underline{n}} M_{\underline{i}}\left(a_{1}, \ldots, a_{2 n}\right) \otimes M_{\underline{i}}^{c}\left(b_{1}, \ldots, b_{2 n}\right) .
\end{aligned}
$$

If $\eta \in S_{2 n}$, then

$$
\begin{aligned}
\left\{a_{\eta(1)} \otimes b_{\eta(1)}, a_{\eta(2)} \otimes b_{\eta(2)}\right\} & \cdots\left\{a_{\eta(2 n-1)} \otimes b_{\eta(2 n-1)}, a_{\eta(2 n)} \otimes b_{\eta(2 n)}\right\} \\
& =\sum_{\underline{i} \subseteq \underline{n}} M_{\underline{i}}\left(a_{\eta(1)}, \ldots, a_{\eta(2 n)}\right) \otimes M_{\underline{i}}^{c}\left(b_{\eta(1)}, \ldots, b_{\eta(2 n)}\right) .
\end{aligned}
$$

Proof of Theorem 9.1. Let

$$
g(x)=g\left(x_{1}, \ldots, x_{2 n}\right)=\sum_{\eta \in T_{2 n}} \gamma_{\eta}\left\{x_{\eta(1)}, x_{\eta(2)}\right\} \cdots\left\{x_{\eta(2 n-1)}, x_{\eta(2 n)}\right\}
$$

be a general multi-linear customary polynomial, with the coefficients $\gamma_{\eta}$ considered as unknowns at the moment. By multi-linearity, $g(x)$ is an identity of $A \otimes B$ if it vanishes for any substitution $x_{u}=a_{u} \otimes b_{u} \in A \otimes B$, for $u=1, \ldots, 2 n$. With such a substitution, by Lemma 9.1

$$
\begin{aligned}
g(a \otimes b) & =g\left(a_{1} \otimes b_{1}, \ldots, a_{2 n} \otimes b_{2 n}\right) \\
& =\sum_{\eta \in T_{2 n}} \gamma_{\eta} \cdot \sum_{\underline{i} \subseteq \underline{n}} M_{\underline{i}}\left(a_{\eta(1)}, \ldots, a_{\eta(2 n)}\right) \otimes M_{\underline{i}}^{c}\left(b_{\eta(1)}, \ldots, b_{\eta(2 n)}\right) \\
& =\sum_{\eta \in T_{2 n}} \gamma_{\eta} \cdot \sum_{\underline{i} \subseteq \underline{n}} M_{\underline{i}}\left(a_{\eta}\right) \otimes M_{\underline{i}}^{c}\left(b_{\eta}\right) .
\end{aligned}
$$

Fix some $\underline{i} \subseteq \underline{n}$ and consider the first products equation in (22): these are customary elements. By definition of customary codimensions, there exist $q_{2|\underline{i}|}(A)$ customary elements such that the first products are expressed via these elements. 
We multiply by the singletons of the second product of (22). Thus, there exist extended customary polynomials $K_{\underline{i}, s}\left(x_{1}, \ldots, x_{2 n}\right), 1 \leq s \leq q_{2|\underline{i}|}(A)$, and coefficients $\alpha_{\underline{i}, s, \eta}$ with $\eta \in T_{2 n}$, such that for any $a_{1}, \ldots, a_{2 n} \in A$,

$$
M_{\underline{i}}\left(a_{\eta}\right)=\sum_{s=1}^{q_{2|\underline{i}|} \mid} \alpha_{\underline{i}, s, \eta} K_{\underline{i}, s}\left(a_{\eta(1)}, \ldots, a_{\eta(2 n)}\right) .
$$

Similarly, for $M_{\underline{i}}^{c}\left(b_{\eta}\right)$, let $\underline{i}^{c}=\underline{n} \backslash \underline{i}$. There exist polynomials $L_{\underline{i}^{c}, t}\left(x_{1}, \ldots, x_{2 n}\right)$, $1 \leq t \leq q_{2\left|\underline{\underline{i}}^{c}\right|}(B)$, and coefficients $\beta_{\underline{i}^{c}, t, \eta}$ with $\eta \in T_{2 n}$, such that for any $b_{1}, \ldots, b_{2 n} \in$ $B$,

$$
M_{\underline{i}}^{c}\left(b_{\eta}\right)=\sum_{t=1}^{q_{2\left|\underline{i}^{c}\right|}(B)} \beta_{\underline{\underline{i}}^{c}, t, \eta} L_{\underline{i}^{c}, t}\left(b_{\eta(1)}, \ldots, b_{\eta(2 n)}\right) .
$$

Thus,

$$
\begin{aligned}
& g(a \otimes b)=\sum_{\eta \in T_{2 n}} \gamma_{\eta} \cdot \sum_{\underline{i} \subseteq \underline{n}} M_{\underline{\underline{i}}}\left(a_{\eta}\right) \otimes M_{\underline{i}}^{c}\left(b_{\eta}\right) \\
& =\sum_{\eta \in T_{2 n}} \gamma_{\eta} \cdot \sum_{\underline{i} \subseteq \underline{n}}\left(\sum_{s=1}^{q_{2|\underline{\underline{i}}|}(A)} \alpha_{\underline{i}, s, \eta} K_{\underline{i}, s}\left(a_{\eta(1)}, \ldots, a_{\eta(2 n)}\right)\right) \\
& \otimes\left(\sum_{t=1}^{q_{2\left|\underline{i}^{c}\right|}(B)} \beta_{\underline{i}^{c}, t, \eta} L_{\underline{i}^{c}, t}\left(b_{\eta(1)}, \ldots, b_{\eta(2 n)}\right)\right) \\
& =\sum_{\underline{i} \subseteq \underline{n}} \sum_{s=1}^{q_{2|\underline{\underline{i}}|}(A)} \sum_{t=1}^{q_{2\left|\underline{\underline{i}}^{c}\right|}(B)}\left[\sum_{\eta \in T_{2 n}} \alpha_{\underline{\underline{i}}, s, \eta} \cdot \beta_{\underline{\underline{\underline{i}}}^{c}, t, \eta} \cdot \gamma_{\eta}\right] \cdot K_{\underline{\underline{i}, s}}\left(a_{\eta}\right) \otimes L_{\underline{\underline{i}}^{c}, t}\left(b_{\eta}\right) \text {. }
\end{aligned}
$$

It follows that $g(x)=0$ is an identity of $A \otimes B$ if for all $\underline{i} \subseteq \underline{n}$, all $1 \leq s \leq q_{2|\underline{\mid}| \underline{\mid}}(A)$, and all $1 \leq t \leq q_{2\left|\underline{i}^{c}\right|}(B)$,

$$
\sum_{\eta \in T_{2 n}} \alpha_{\underline{i}, s, \eta} \cdot \beta_{\underline{\underline{i}}^{c}, t, \eta} \cdot \gamma_{\eta}=0 .
$$

This is a system of linear equations in $\left|T_{2 n}\right|$ unknowns $\gamma_{\eta}$. Set $a=|\underline{i}|$ and $b=\left|\underline{i}^{c}\right|$; then $a+b=n$. The number of equations is given by

$$
\sum_{a+b=n}\left(\begin{array}{l}
n \\
a
\end{array}\right) q_{2 a}(A) \cdot q_{2 b}(B) .
$$

By Theorem 8.1 the customary codimensions $q_{2 n}(A)$ and $q_{2 n}(B)$ are exponentially bounded. Hence, the number of equations is exponentially bounded. On the other hand, $\left|T_{2 n}\right|$ grows over-exponentially; see equation (13). If $n$ is large enough, this system has a nontrivial solution, namely a nontrivial $g(x)$ which is an identity of $A \otimes B$.

Corollary 9.1. Let $A$ and $B$ be two Poisson PI algebras. Then we have the following estimate on the customary exponent of $A \otimes B$ :

$$
\operatorname{Exp}^{Q}(A \otimes B) \leq \operatorname{Exp}^{Q} A+\operatorname{Exp}^{Q} B .
$$


Proof. For simplicity, suppose that $q_{2 a}(A) \leq \lambda^{2 a}$ and $q_{2 b}(B) \leq \mu^{2 b}$ for all $a, b$. Remark that the number of equations (23) yields the upper bound on the customary codimension growth of $A \otimes B$. We get

$$
\begin{aligned}
q_{2 n}(A \otimes B) \leq \sum_{a+b=n}\left(\begin{array}{l}
n \\
a
\end{array}\right) q_{2 a}(A) \cdot q_{2 b}(B) \leq \sum_{a+b=n}\left(\begin{array}{l}
n \\
a
\end{array}\right) \lambda^{2 a} \mu^{2 b} \\
\leq \sum_{a+b=n}\left(\begin{array}{c}
2 n \\
2 a
\end{array}\right) \lambda^{2 a} \mu^{2 b} \leq \sum_{i+j=2 n}\left(\begin{array}{c}
2 n \\
i
\end{array}\right) \lambda^{i} \mu^{j}=(\lambda+\mu)^{2 n} .
\end{aligned}
$$

We conclude by noting that this estimate is exact for Hamiltonian algebras; see Corollary 5.2 and (21).

\section{REFERENCES}

[1] Andrews G.E., The theory of partitions, Addison-Wesley, 1976. MR0557013 (58:27738)

[2] Bahturin Yu. A., Identical relations in Lie algebras. VNU Science Press, Utrecht, 1987. MR0886063 (88f:17032)

[3] Bahturin Yu., Mishchenko S., Regev A., On the Lie and associative codimensions growth. Comm. Algebra 27 (1999), no. 10, 4901-4908. MR.1709214 (2000m:16034)

[4] Berele A., Regev A., Hook Young diagrams with applications to combinatorics and to representations of Lie superalgebras, Adv. Math. 64 (1987), no. 2, 118-175. MR0884183 (88i:20006)

[5] Dixmier J., Enveloping algebras. AMS, Rhode Island, 1996. MR1393197(97c:17010)

[6] Drensky V., Identities of representations of nilpotent Lie algebras. Comm. Algebra 25 (1997), no. 7, 2115-2127. MR 1451682 (98d:17015)

[7] Drensky V., Free algebras and PI-algebras. Graduate course in algebra. Springer-Verlag Singapore, Singapore, 2000. MR 1712064 (2000j:16002)

[8] Farkas D. R., Poisson polynomial identities. Comm. Algebra 26 (1998), no. 2, 401-416. MR1603345 (98m:16029)

[9] Farkas D. R., Poisson polynomial identities. II. Arch. Math. (Basel) 72 (1999), no. 4, 252-260. MR1678045 (2000d:16034)

[10] Giambruno A., Zaicev M., Exponential codimension growth of PI algebras: an exact estimate. Adv. Math. 142 (1999), no. 2, 221-243. MR1680198 (2000a:16048)

[11] Kac V. G., Simple irreducible graded Lie algebras of finite growth. Izv. Akad. Nauk SSSR Ser. Mat. 32 (1968) 1323-1367. MR0259961 (41:4590)

[12] Macdonald I. G., Symmetric functions and Hall polynomials, 2-nd ed., Oxford: Clarendon Press, 1995. MR.1354144 (96h:05207)

[13] Mathieu O., Classification of simple graded Lie algebras of finite growth. Invent. Math. 108 (1992), no. 3, 455-519. MR:1163236 (93h:17069)

[14] Mischchenko S.P., Regev, A., Zaicev M., Integrality of exponents of some abelian-bynilpotent varieties of Lie algebras. Comm. Algebra, 28 (2000), no. 9, 4105-4130. MR1772005 (2001g:17019)

[15] Petrogradsky V.M., On complexity functions for T-ideals of associative algebras. Mat. Zametki 68 (2000), no. 6, 887-897; translation in Math. Notes 68 (2000), no. 5-6, 751-759. MR:1835188 (2002c:16031)

[16] Razmyslov Yu. P., Identities of Algebras and Their representations, A.M.S Translations of Math Monographs Vol 138 (1994). MR1291603 (95i:16022)

[17] Regev A. Existence of identities in $A \otimes B$. Israel J. Math. 11 (1972), 131-152. MR0314893 (47:3442)

[18] Shestakov I.P., Quantization of Poisson superalgebras and speciality of Jordan Poisson superalgebras. Algebra i Logika. 32 (1993), no. 5, 571-584; English translation: Algebra and Logic, 32 (1993), no. 5, 309-317. MR1287006 (95c:17034)

[19] Stanley R. P., Enumerative combinatorics, Vol. 2. Cambridge University Press, Cambridge, 1999. MR.1676282(2000k:05026) 
[20] Tarasov A.A., On uniqueness of raising of maximal commutative subalgebras in Poisson algebras into universal enveloping algebra. Mat. Sbornik. 194 (2003), No. 7, 155-160. MR2020383 (2004k:17023)

[21] Volichenko I. B., Varieties of Lie algebras with identity $\left[\left[X_{1}, X_{2}, X_{3}\right],\left[X_{4}, X_{5}, X_{6}\right]\right]=0$ over a field of characteristic zero. Sibirsk. Mat. Zh. 25 (1984), no. 3, 40-54. MR 0746940 (85k:17016)

Faculty of Mathematics, Ulyanovsk State University, Leo Tolstoy 42, Ulyanovsk, 432970 RUSSIA

E-mail address: mishchenkosp@mail.ru

E-mail address: mishchenkosp@ulsu.ru

Faculty of Mathematics, Ulyanovsk State University, Leo Tolstoy 42, Ulyanovsk, 432970 Russia

E-mail address: petrogradsky@hotbox.ru

Department of Theoretical Mathematics, Weizmann Institute of Science, Rehovot, ISRAEL

E-mail address: regev@wisdom.weizmann.ac.il 